\title{
X-ray bursts at extreme mass accretion rates from $\mathrm{GX} 17+2$
}

\author{
E. Kuulkers ${ }^{1,2}$, J. Homan ${ }^{3, \star}$, M. van der Klis ${ }^{3}$, W. H. G. Lewin ${ }^{4}$, and M. Méndez ${ }^{1}$ \\ 1 SRON National Institute for Space Research, Sorbonnelaan 2, 3584 CA Utrecht, The Netherlands \\ e-mail: M.Mendez@sron.nl \\ 2 Astronomical Institute, Utrecht University, PO Box 80000, 3508 TA Utrecht, The Netherlands \\ 3 Astronomical Institute "Anton Pannekoek", University of Amsterdam, and Center for High Energy \\ Astrophysics, Kruislaan 403, 1098 SJ Amsterdam, The Netherlands \\ e-mail: homan@merate.mi.astro.it; michiel@astro.uva.nl \\ 4 Department of Physics and Center for Space Research, Massachusetts Institute of Technology, Cambridge, \\ MA 02138, USA \\ e-mail: lewin@space.mit.edu
}

Received 23 May 2001 / Accepted 15 November 2001

\begin{abstract}
We report on ten type I X-ray bursts originating from GX $17+2$ in data obtained with the RXTE/PCA in 1996-2000. Three bursts were short in duration $(\sim 10 \mathrm{~s})$, whereas the others lasted for $\sim 6-25$ min. All bursts showed spectral softening during their decay. There is no evidence for high-frequency $(>100 \mathrm{~Hz})$ oscillations at any phase of the bursts. We see no correlations of the burst properties with respect to the persistent X-ray spectral properties, suggesting that in GX $17+2$ the properties of the bursts do not correlate with inferred mass accretion rate. The presence of short bursts in GX $17+2$ (and similar bright X-ray sources) is not accounted for in the current $\mathrm{X}$-ray bursts theories at the high mass accretion rates encountered in this source. We obtain satisfactory results if we model the burst emission with a black body, after subtraction of the persistent pre-burst emission. The twocomponent spectral model does not fit the total burst emission whenever there is a black-body component present in the persistent emission. We conclude that in those cases the black-body contribution from the persistent emission is also present during the burst. This implies that, contrary to previous suggestions, the burst emission does not arise from the same site as the persistent black-body emission. The black-body component of the persistent emission is consistent with being produced in an expanded boundary layer, as indicated by recent theoretical work. Five of the long bursts showed evidence of radius expansion of the neutron star photosphere (independent of the spectral analysis method used), presumably due to the burst luminosity reaching the Eddington value. When the burst luminosity is close to the Eddington value, slight deviations from pure black-body radiation are seen at energies below $\simeq 10 \mathrm{keV}$. Similar deviations have been seen during (long) X-ray bursts from other sources; they can not be explained by spectral hardening models. The total persistent flux just before and after the radius expansion bursts is inferred to be up to a factor of 2 higher than the net peak flux of the burst. If both the burst and persistent emission are radiated isotropically, this would imply that the persistent emission is up to a factor of 2 higher than the Eddington luminosity. This is unlikely and we suggest that the persistent luminosity is close to the Eddington luminosity and that the burst emission is (highly) anisotropic $(\xi \sim 2)$. Assuming that the net burst peak fluxes equal the Eddington limit, applying standard burst parameters $\left(1.4 M_{\odot}\right.$ neutron star, cosmic composition, electron scattering opacity appropriate for high temperatures), and taking into account gravitational redshift and spectral hardening, we derive a distance to GX $17+2$ of $\sim 8 \mathrm{kpc}$, with an uncertainty of up to $\sim 30 \%$.
\end{abstract}

Key words. accretion, accretion disks - binaries: close - stars: individual (GX 17+2) - stars: neutron X-rays: bursts

\section{Introduction}

X-ray bursts were discovered in 1975 from the source 4U 1820-30 (Grindlay \& Heise 1975; Grindlay et al. 1976). It was realized soon thereafter that these were thermo-nuclear runaway events on the surface of neutron

Send offprint requests to: E. Kuulkers at SRON, e-mail: E.Kuulkers@sron.nl

* Present address: Osservatorio Astronomico di Brera, Via E. Bianchi 46, 23807 Merate (LC), Italy. stars (Woosley \& Taam 1976; Maraschi \& Cavaliere 1977). Another kind of X-ray bursts was found (together with the above type of bursts) from MXB 1730-355 (later referred to as the Rapid Burster), which were suggested to be due to accretion instabilities. The former and latter kind of bursts were then dubbed types I and II, respectively (Hoffman et al. 1978a).

The main characteristics of type I bursts (for a review see Lewin et al. 1993) are: sudden and short $(\simeq 1 \mathrm{~s})$ increase in the X-ray flux, exponential decay light curve, duration 
of the order of seconds to minutes, softening during the decay (attributed to cooling of the neutron star surface), (net) burst spectra reasonably well described by blackbody emission from a compact object with $\simeq 10 \mathrm{~km}$ radius and temperature of $\simeq 1-2 \mathrm{keV}$, and total energies ranging from $\simeq 10^{39}$ to $10^{40} \mathrm{erg}$. When the luminosity during the burst reaches the Eddington limit (i.e., when the pressure force due to radiation balances the gravitational force), the neutron star photosphere expands. Since $L_{\mathrm{b}} \propto R^{2} T_{\mathrm{eff}}^{4}$, when the radius of the photosphere, $R$, expands, the effective temperature, $T_{\text {eff }}$, drops, with the burst luminosity, $L_{\mathrm{b}}$, being constant (modulo gravitational redshift effects with changing $R$ ) at the Eddington limit, $L_{\mathrm{Edd}}$. Bursts during their radius expansion/contraction phase are therefore recognizable by an increase in the inferred radius with a simultaneous decrease in the observed temperature, while the observed flux stays relatively constant.

The emission from a (hot) neutron star is not expected to be perfectly Planckian, however (van Paradijs 1982; London et al. 1984, 1986; see also Titarchuk 1994; Madej 1997, and references therein). This is mainly due to the effects of electron scattering in the neutron star atmosphere, deforming the original X-ray spectrum. This results in a systematic difference between the effective temperature (as would be measured on Earth), $T_{\text {eff, } \infty}$, and the temperature as obtained from the spectral fits, $T_{\mathrm{bb}}$ (also referred to as "colour" temperature, see e.g. Lewin et al. 1993). In general, the deviations from a Planckian distribution will depend on several parameters, such as temperature, elemental abundance, neutron star mass and radius. The hardening factor, $T_{\mathrm{bb}} / T_{\mathrm{eff}, \infty}$, has been determined through numerical calculations by various people and its value is typically around 1.7. When the burst luminosity approaches the Eddington limit the deviations from a black-body become larger, and so does the spectral hardening $\left(T_{\mathrm{bb}} / T_{\text {eff, } \infty} \sim 2\right.$, Babul \& Paczyński 1987; Titarchuk 1988). During extreme radius expansion phases, however, this trend may break down and $T_{\mathrm{bb}} / T_{\mathrm{eff}, \infty}<1$ (Titarchuk 1994). Attempts have been made to determine the spectral hardening from the observed cooling tracks, but conclusions are still rather uncertain (e.g. Penninx et al. 1989; Smale 2001). As a result, the interpretation of X-ray bursts spectra has remained uncertain and constraints on the mass-radius relationship for neutron stars elusive.

Type I X-ray burst theory predicts three different regimes in mass accretion rate $(\dot{M})$ for unstable burning (Fujimoto et al. 1981; Fushiki \& Lamb 1987; see also Bildsten 1998, 2000; Schatz et al. 1999, and references therein; note that values of critical $\dot{M}$ depend on metallicity, and on assumed core temperature and mass of the neutron star):

1) low accretion rates; $10^{-14} M_{\odot} \mathrm{yr}^{-1} \lesssim \dot{M} \lesssim 2 \times$ $10^{-10} M_{\odot} \mathrm{yr}^{-1}$ : mixed $\mathrm{H} / \mathrm{He}$ burning triggered by thermally unstable $\mathrm{H}$ ignition;
2) intermediate accretion rates; $2 \times 10^{-10} M_{\odot} \mathrm{yr}^{-1} \lesssim$ $\dot{M} \lesssim 4-11 \times 10^{-10} M_{\odot} \mathrm{yr}^{-1}$ : pure He shell ignition after steady $\mathrm{H}$ burning;

3) high accretion rates; $4-11 \times 10^{-10} M_{\odot} \mathrm{yr}^{-1} \lesssim \dot{M} \lesssim$ $2 \times 10^{-8} M_{\odot} \mathrm{yr}^{-1}$ : mixed $\mathrm{H} / \mathrm{He}$ burning triggered by thermally unstable He ignition.

$\mathrm{H}$ and $\mathrm{He}$ are burning stably in a mixed $\mathrm{H} / \mathrm{He}$ environment for very low and very high values of $\dot{M}$, i.e. $\dot{M}$ below $\sim 10^{-14} M_{\odot} \mathrm{yr}^{-1}$ and above $\sim 2 \times 10^{-8} M_{\odot} \mathrm{yr}^{-1}$ (close to the critical Eddington $\dot{M})$. During pure helium flashes the fuel is burned rapidly, and such bursts therefore last only $5-10 \mathrm{~s}$. This gives rise to a large energy release in a short time, which causes the bursts often to reach the Eddington limit, leading to photospheric radius expansion. Bursts with unstable mixed $\mathrm{H} / \mathrm{He}$ burning release their energies on a longer, 10-100s, timescale, due to the long series of $\beta$ decays in the rp-process (see e.g. Bildsten 1998, 2000).

The Z sources (Hasinger \& van der Klis 1989) are a group of sources inferred to persistently accrete near the Eddington limit. In a colour-colour diagram they trace out a Z-like shape, with the three limbs of the Z (historically) referred to as the horizontal branch (HB), normal branch (NB) and flaring branch (FB), from top to bottom. $\dot{M}$ is inferred to increase from sub-Eddington at the $\mathrm{HB}$, near-Eddington at the NB to super-Eddington at the FB (e.g. Hasinger 1987; Lamb 1989; Hasinger et al. 1990). According to the burning regimes outlined above these sources should exhibit long ( $210-100 \mathrm{~s})$ type I Xray bursts, at least on the $\mathrm{HB}$ and NB. However, of the Z sources, only GX $17+2$ and Cyg X-2 show (infrequent) bursts (Kahn \& Grindlay 1984; Tawara et al. 1984c; Sztajno et al. 1986; Kuulkers et al. 1995, 1997; Wijnands et al. 1997; Smale 1998), indicating that most of the material is burning stably. This is in contrast to the above theoretical expectations for high $\dot{M}$. Moreover, the bursts in Cyg X-2 are short ( $\simeq 5 \mathrm{~s})$, whereas GX $17+2$ shows both short $(\simeq 10 \mathrm{~s})$ and long $(\gtrsim 100 \mathrm{~s})$ bursts. One of the bursts of Cyg X-2 showed a radius expansion phase (Smale 1998); this burst clearly bears all the characteristics of a He flash (regime 2), whereas the neutron star is inferred to accrete at near-Eddington rates. The short duration bursts in GX $17+2$ also hint to a He flash origin, whereas the long duration bursts hint to unstable mixed H/He burning (regime 3; see also van Paradijs et al. 1988). A study of X-ray bursts from Cyg X-2 and GX 17+2 observed by EXOSAT showed no correlation of the burst properties with position in the $\mathrm{Z}$, although the number of bursts observed from GX 17+2 was small (Kuulkers et al. 1995, 1997).

The Proportional Counter Array (PCA) onboard the Rossi X-ray Timing Explorer (RXTE) combines high throughput using a large collecting area (maximum of $\simeq 6500 \mathrm{~cm}^{2}$ ) with the ability to label photons down to a time resolution of $\mu \mathrm{s}$. This is ideal to study short events like X-ray bursts, especially during the start of the burst. Such studies may provide more insight in the properties 
Table 1. RXTE observation log of GX $17+2^{a}$.

\begin{tabular}{lllllllcc}
\hline Year & \multicolumn{2}{c}{ Start (UT) } & \multicolumn{2}{c}{ End (UT) } & $\begin{array}{c}t_{\exp }{ }^{b} \\
\text { ksec) }\end{array}$ & $\begin{array}{c}\text { \# of } \\
\text { bursts }\end{array}$ \\
\hline 1996 & Feb. & 07 & $13: 27$ & Feb. & 09 & $00: 02$ & 58 & 1 \\
1997 & Feb. & 02 & $19: 13$ & Feb. & 27 & $03: 34$ & 59 & 1 \\
1997 & Apr. & 01 & $19: 13$ & Apr. & 04 & $23: 26$ & 35 & 0 \\
1997 & Jul. & 27 & $02: 13$ & Jul. & 28 & $00: 33$ & 43 & 0 \\
1998 & Aug. & 07 & $06: 40$ & Aug. & 08 & $23: 40$ & 71 & 1 \\
1998 & Nov. & 18 & $06: 42$ & Nov. & 20 & $13: 31$ & 86 & 2 \\
1999 & Oct. & 03 & $02: 43$ & Oct. & 12 & $07: 05$ & 298 & 5 \\
2000 & Mar. & 31 & $12: 15$ & Mar. & 31 & $16: 31$ & 7 & 0 \\
\hline
\end{tabular}

${ }^{a}$ Between Feb. 2-27, 1997 observations every 3-6 days.

${ }^{b}$ Total effective exposure time.

of X-ray bursts which occur at these extreme mass accretion rates. Analysis of X-ray bursts in persistent sources at (relatively) high inferred mass accretion rates (typically $\gtrsim 0.2 \dot{M}_{\text {Edd }}$ ) observed with the RXTE/PCA were presented for one burst seen with Cyg X-2 (Smale 1998) and one seen in GX 3+1 (Kuulkers \& van der Klis 2000). In this paper we present the first account of ten X-ray bursts from GX $17+2$ observed by the RXTE/PCA during the period 1996-2000. For a description of the correlated Xray timing and spectral properties of GX $17+2$ using the same data set we refer to Homan et al. (2001).

\section{Observations and analysis}

The PCA (2-60 keV; Bradt et al. 1993) onboard RXTE observed GX $17+2$ various times during the mission. Up to now a total of $657 \mathrm{ksec}$ of useful data has been obtained. A $\log$ of these observations is given in Table 1. During the observations in 1996-1998 all five proportional counter units (PCUs) were active, whereas in 1999 and 2000 only three units were active. The high voltage settings of the PCUs have been altered three times during the mission (so-called gain changes), which modified the response of the detectors. These changes therefore mark four periods, called gain epochs. The observations were done in two standard modes: one with relatively high spectral resolution (129 energy channels covering the whole PCA energy band) every $16 \mathrm{sec}$, the STANDARD 2 mode, the other having no spectral information providing the intensity in the whole PCA energy band at a moderate time resolution of $0.125 \mathrm{~s}$, the STANDARD 1 mode. Additionally, data were recorded in various high time resolution $(\leq 2 \mathrm{~ms})$ modes that ran in parallel to the STANDARD modes, and that recorded photons within a specific energy band with either low spectral resolution (B-modes or E-modes) or no spectral resolution (SB-modes). The B-, E- and SB-modes used here combined the information from all layers of all active PCUs together. During the 1996 observations a Band E-mode were available, giving 16 and 64 energy bands, covering channels $0-49$ and 50-249, at $2 \mathrm{~ms}$ and $125 \mu \mathrm{s}$, respectively. For most of the observations in 1997 four SB modes covering the total PCA energy range were available. In the 1998, 1999, and 2000 observations two SB-modes covering channels $0-13$ and $14-17$ at $125 \mu \mathrm{s}$, and an Emode giving 64 energy bands covering channels 18-249 at $16 \mu$ s time resolution were available.

For the spectral analysis of the persistent emission we used the STANDARD 2 data. We accumulated data stretches of $96 \mathrm{~s}$ just before the burst, combining the PCUs which were operating at that time. In order to study the spectral properties of the bursts we used two approaches. Spectra during the bursts were determined every $0.25 \mathrm{~s}$ for the first $\simeq 20$ s of the burst. For the short bursts this means the whole duration of the burst. For the long bursts we also used the STANDARD 2 data to create spectra at $16 \mathrm{~s}$ intervals, for evaluating the remainder of these bursts. Since no high time resolution spectral data were available during the 1997 observations (only 4 SB-modes), only the STANDARD 2 data were used to study the spectral properties of the burst from this observation. All spectra were corrected for background and dead-time using the procedures supplied by the RXTE Guest Observer Facility ${ }^{1}$. A systematic uncertainty of $1 \%$ in the count rate spectra was taken into account. For our spectral fits we confined ourselves to the energy range of $3-20 \mathrm{keV}$, which is best calibrated. The hydrogen column density, $N_{\mathrm{H}}$, towards GX $17+2$ was fixed to that found by the Einstein SSS and MPC measurements $\left(2 \times 10^{22}\right.$ atoms cm ${ }^{-2}$, Christian \& Swank 1997; see also Di Salvo et al. 2000). In all cases we included a Gaussian line (see Di Salvo et al. 2000) fixed at $6.7 \mathrm{keV}$, with a fixed line width of $0.1 \mathrm{keV}$. One sigma confidence errors were determined using $\Delta \chi^{2}=1$.

Large amplitude, high coherence brightness oscillations have been observed during various type I X-ray bursts in other low-mass X-ray binaries (LMXBs; see e.g. Strohmayer 1998, 2001). We searched all the bursts from GX $17+2$ for such oscillations. Using the high time resolution modes we performed Fast Fourier Transforms to produce power spectra with a Nyquist frequency of $2048 \mathrm{~Hz}$. This was done in the total energy band (2-60 keV) and in a high energy band ranging from $\simeq 8 \mathrm{keV}$ to $\simeq 20 \mathrm{keV}$. For the 1996 observations, however, the high time resolution mode only covered the $13.5-60 \mathrm{keV}$ range; the $2-60 \mathrm{keV}$ power spectra had a Nyquist frequency of $256 \mathrm{~Hz}$. In all cases, our searches were carried out on power spectra of $0.25 \mathrm{~s}$ or $2 \mathrm{~s}$ in duration. To increase the sensitivity for cases where burst oscillations are only present for a period of time comparable or shorter than the length of the power spectrum, we "oversampled" the data by factors of 2 and 8, respectively, by taking the start time of the next data segment to be $0.125 \mathrm{~s}$ and $0.25 \mathrm{~s}$ later than that of the previous one, instead of $0.25 \mathrm{~s}$ and $2 \mathrm{~s}$ (i.e. we use overlapping data segments).

\footnotetext{
1 http://heasarc.gsfc.nasa.gov/docs/xte/recipes/ cook_book.html.
} 
Table 2. Bursts and burst-like events (flares) in GX 17+2.

\begin{tabular}{|c|c|c|c|c|c|c|c|c|c|c|c|c|}
\hline event $^{a}$ & & tart T & me & UT) & $E^{b}$ & $\mathrm{PCUs}^{c}$ & $t_{\mathrm{dur}}{ }^{d}$ & $t_{\text {rise }}{ }^{e}$ & $t_{\mathrm{fr}}{ }^{f}$ & $t_{\exp }^{g}$ & $\chi_{\text {red }}^{2} /$ d.o.f. $^{h}$ & branch $^{i}$ \\
\hline b1 & 1996 & Feb. & 08 & $16: 17: 12$ & 1 & 5 & 10 & 1.22 & $0.35 \pm 0.05$ & $1.83 \pm 0.08$ & $1.1 / 130$ & $\mathrm{mNB}$ \\
\hline b2 & 1997 & Feb. & 08 & $02: 36: 34$ & 3 & 5 & $>360$ & 1.19 & $0.34 \pm 0.08$ & $248+4$ & $2.5 / 49$ & $\mathrm{mNB}$ \\
\hline b3 & 1998 & Aug. & 07 & $13: 15: 50$ & 3 & 5 & 10 & 0.53 & $0.27 \pm 0.09$ & $2.55 \pm 0.24$ & $1.0 / 131$ & INB \\
\hline $\mathrm{b} 4$ & 1998 & Nov. & 18 & $08: 51: 26$ & 3 & 5 & 1000 & 1.34 & $0.61 \pm 0.04$ & $197 \pm 2$ & $3.7 / 147$ & SV \\
\hline b5 & 1998 & Nov. & 18 & $14: 37: 30$ & 3 & 5 & 10 & 0.41 & $0.54 \pm 0.04$ & $2.06 \pm 0.13$ & $1.1 / 85$ & $\mathrm{mNB}$ \\
\hline b6 & 1999 & Oct. & 03 & $15: 36: 32$ & 4 & $3(0,2,3)$ & 1600 & 0.41 & $0.19 \pm 0.04$ & $274 \pm 3$ & $2.0 / 242$ & lHB \\
\hline b7 & 1999 & Oct. & 05 & $23: 41: 43$ & 4 & $3(0,2,4)$ & 500 & 0.56 & $0.30 \pm 0.03$ & $77.3 \pm 1.2$ & $1.9 / 104$ & uNB \\
\hline b8 & 1999 & Oct. & 06 & $11: 10: 33$ & 4 & $3(0,2,3)$ & 500 & 1.66 & $0.13 \pm 0.02$ & $70.2 \pm 1.4$ & $1.2 / 57$ & lHB \\
\hline b9 & 1999 & Oct. & 09 & $12: 34: 24$ & 4 & $3(0,2,3)$ & 500 & 0.13 & $0.16 \pm 0.02$ & $76.4 \pm 1.5$ & $3.1 / 66$ & uNB \\
\hline b10 & 1999 & Oct. & 10 & 09:10:47 & 4 & $3(0,2,3)$ & 700 & 0.72 & $0.20 \pm 0.04$ & $115 \pm 3$ & $3.7 / 57$ & lNB \\
\hline f1 & 1996 & Feb. & 07 & 03:39:11 & 1 & 5 & 10 & 1.03 & $1.12 \pm 0.07$ & $2.98 \pm 0.49$ & $1.3 / 88$ & $\mathrm{mFB}$ \\
\hline $\mathrm{f} 2$ & 1998 & Nov. & 19 & $14: 38: 24$ & 3 & 5 & 10 & 0.41 & $0.33 \pm 0.04$ & $1.72 \pm 0.35$ & $1.1 / 28$ & $\mathrm{uFB}$ \\
\hline f3 & 1998 & Nov. & 20 & $00: 44: 43$ & 3 & 5 & 10 & 0.75 & $0.45 \pm 0.06$ & $1.85 \pm 0.25$ & $1.1 / 55$ & $\mathrm{uFB}$ \\
\hline f4 & 1999 & Oct. & 11 & 08:55:30 & 4 & $3(0,2,3)$ & 10 & 5.0 & $1.06 \pm 0.20$ & $2.18 \pm 0.22$ & $1.1 / 148$ & $\mathrm{lFB}$ \\
\hline
\end{tabular}

${ }^{a}$ Designation used in text.

${ }^{b}$ RXTE gain epoch of the observation.

${ }^{c}$ Numbers of active PCUs; if $<5$ the PCU unit numbers are given between brackets.

${ }^{d}$ Approximate duration in $\mathrm{s}$.

${ }^{e}$ Time (s) for the count rate to rise from $25 \%$ to $90 \%$ of the net-peak rate.

${ }^{f}$ Duration of fast rise phase in s, see text.

${ }^{g}$ Exponential decay time in s.

${ }^{h}$ Goodness of the exponential fit to the decay part of the light curve.

${ }^{i}$ Source position in the $\mathrm{Z}$ just before the event (see also Fig. 16); u, m, and 1 stand for upper, middle and lower, respectively. SV means "soft" vertex, i.e. the NB/FB transition region.

\section{Results}

\subsection{Temporal behaviour}

Inspection of all the STANDARD 1 light curves of GX $17+2$ at $1 \mathrm{~s}$ time resolution yielded a total of fourteen burst-like events (designated b1, b2, ..., b10, f1, ..., f4; the reason for different designations is the nature of the events, see below). Seven events were rather short, $\simeq 10 \mathrm{~s}$ (b1, b3, b5, f1-f4), whereas the other events were long, $\simeq 360-1600$ s (b2, b4, b6-b10). Table 2 gives the start times of these events, together with the gain epoch in which the observations were done and the number of active PCUs. In that table we also give the duration of the events as estimated by eye using the light curves in the total PCA energy band and/or the hardness (ratio of count rates in two energy bands) curves, the rise time of the event as determined in two ways (see below), and the exponential decay time as determined from a fit to the decay part of the STANDARD 1 light curves, together with the goodness of this fit expressed in terms of reduced $\chi^{2}, \chi_{\text {red }}^{2}$. We note that the estimates of the durations are rather arbitrary, but robust methods such as the so-called T90 measurements $^{2}$ as used for $\gamma$-ray bursts (see Koshut et al. 1996,

\footnotetext{
${ }^{2}$ T90 is defined as the time it takes to observe $90 \%$ of the total background-subtracted counts in an event, starting and
}

and references therein) can not be applied, especially to the longer events, since the persistent emission varies on the same time scale (or even fatser) as the event itself, see e.g. Fig. 1. The rise time of the events was determined as follows. We constructed light curves in the full PCA energy band with a time resolution of $1 / 32 \mathrm{~s}$. We defined $t_{\text {rise }}$ as the time for the event to increase from $25 \%$ to $90 \%$ of the net peak event rate (see e.g. Muno et al. 2000; van Straaten et al. 2001). A detailed look at the light curves revealed that the onset of events b1-b4, b8, b10, and $\mathrm{f} 2$ consisted of a rapid rise phase and a subsequent slower rise to maximum. In the events b5-b7, b9, f1, and f3 the count rate rapidly increased to maximum, with no subsequent slower rise. We therefore also fitted the preevent phase, fast rise phase (and slow rise phase) with a constant level and one (or two) linear functions, respectively, using the STANDARD 1 light curves. We find that the total duration of the fast rise phase, $t_{\mathrm{fr}}$, is between 0.1 and $0.6 \mathrm{~s}$ for events b1-b10 and $0.4-1 \mathrm{~s}$ for $\mathrm{f} 1-\mathrm{f} 4$ (Table 2 ). In the fits to the decay portion of the light curves for the short events we used the STANDARD 1 light curves at $0.125 \mathrm{~s}$ time resolution, while for the longer events we rebinned these light curves to a time resolution of $5 \mathrm{~s}$. For

ending when $5 \%$ and $95 \%$ of the total background-subtracted counts have been observed. 


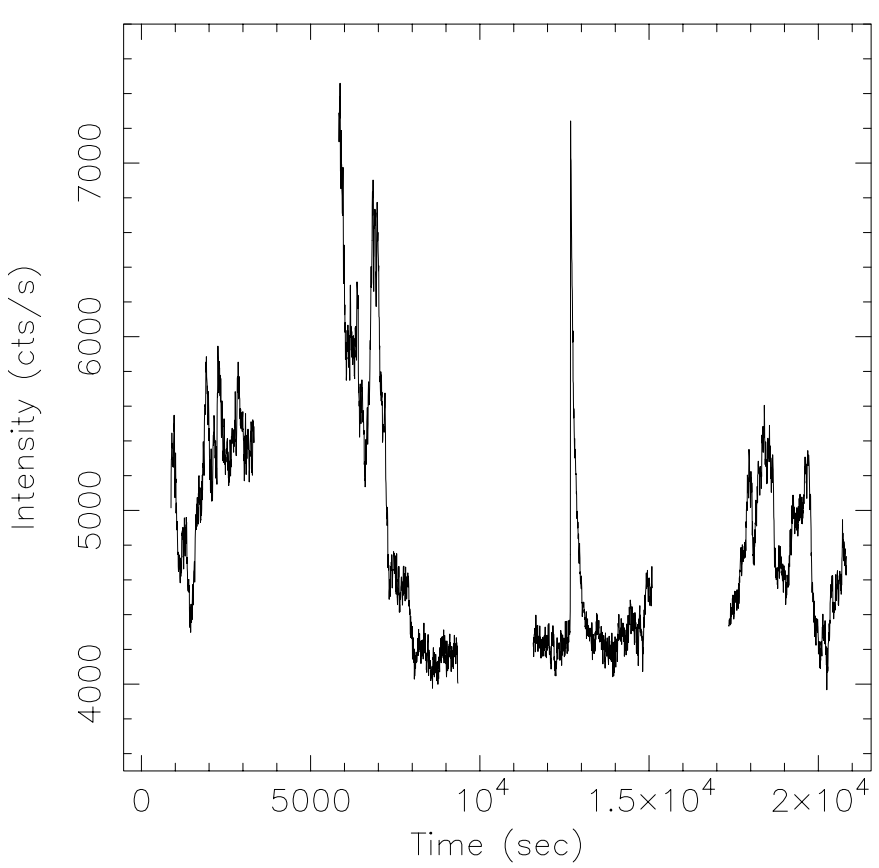

Fig. 1. StAndard 1 light curve of the GX $17+2$ observations during October 10, 1999, at a time resolution of $5 \mathrm{~s}$. Time zero corresponds to 05:39:27 (UTC). No corrections for background and dead-time have been applied. Clearly, the source varies on the same time scale (and faster) as the burst which started at $T=12680 \mathrm{~s}$. The source was in the FB and the lower part of the NB during the observations.

some of the events an exponential does not describe the decay very well; this is probably due to the short term variations in the persistent emission. For these events we fitted only the initial decay (first few seconds for f2 and f3, and first few $100 \mathrm{~s}$ for burst b9 and b10).

In Fig. 2 we show the light curves of the four events f1-f4, at low $(\lesssim 7 \mathrm{keV})$ and high $(\gtrsim 7 \mathrm{keV})$ energies, with the corresponding hardness (ratio of the count rates in the high and low energy band) curves, all at a time resolution of $0.125 \mathrm{~s}$. Although they have a fast rise and a longer decay (see also Table 2), they show small or no variations in hardness. Time-resolved spectral analysis (like done in Sect. 3.2.3) confirmed this; no clear cooling during the decay can be discerned. We can therefore not classify these events as type I bursts. Since all of the four events occurred in the FB, we conclude that they must be flares, of which the light curves happen to resemble those of X-ray bursts (such as b1, b3 and b5). We will not discuss these four events further in the paper and denote the remainder of the ten events as bursts, since we will show below that they are genuine type I X-ray bursts.

In Fig. 3 we show the light curves of the three short bursts b1, b3 and b5, at low and high energies, with the corresponding hardness curves, all at a time resolution of $0.125 \mathrm{~s}$. All three bursts show a fast rise (typically less than $0.5 \mathrm{~s}$ ) and an exponential decay with a decay time of $\simeq 2 \mathrm{~s}$ (see Table 2). During the rise the emission hardens; as the bursts decay, the emission becomes softer. The main difference between the three bursts is the fact that the peak of burst b3 is about $25 \%$ lower than the other two bursts; it looks like a "failed" burst. It also has two peaks, as if some new unstable burning occurred, $\simeq 5 \mathrm{~s}$ after the start of the burst.

In Figs. 4 and 5 we show the light curves of the long bursts b2, b4, and b6-b10, at low and high energies, with the corresponding hardness curves, all at a time resolution of 2 s. In Figs. 6 and 7 we focus on the start of these bursts, all at a time resolution of $0.125 \mathrm{~s}$. Again the rise times are very short (also typically less than $0.5 \mathrm{~s}$ ), but the decay times are much longer, with decay times in the range $\simeq 70-280$ s (see Table 2 ). Apart from the fact that the hard burst emission decays faster than the soft burst emission (i.e. spectral softening), there are more pronounced differences between the light curves in the two energy bands. In Figs. 4 and 5 one sees that all the low energy light curves show a kind of spike at the start of the decay. These spikes last for a few seconds in most cases; however, for burst b6 it seems to last $\simeq 15 \mathrm{~s}$ (with an exponential decay time of $9 \pm 2 \mathrm{~s}$ ). At high energies no such spikes occur (except for burst b10); instead the bursts have more flattopped peaks, with durations ranging from tens of seconds to $\simeq 200$ s. Burst b6 is the nicest example of this. Zooming in on the start of these long bursts, it becomes clear that the rise is somewhat slower at high energies with respect to low energies (causing the hardening of the emission during the early phase of the burst). Also, in bursts b4 and b6-b9 very short $(<0.5 \mathrm{~s})$ spikes occur during the rise in the high energy light curve, which again are especially evident in burst b6 (two spikes!). This causes the hardness to drop on the same time scale in some of these bursts. Later we will show that this corresponds to fast radius expansion/contraction episodes.

\subsection{Spectral behaviour}

\subsubsection{Persistent emission before the bursts}

The persistent emission just before the bursts b6 and b7 can be satisfactorily $\left(\chi_{\text {red }}^{2}=0.7\right.$ and 1.2 , respectively, for 35 degrees of freedom, d.o.f.) described by an absorbed cut-off power-law component plus a Gaussian line. For the persistent emission before the remainder of the bursts this is not a satisfactory model $\left(\chi_{\text {red }}^{2}\right.$ ranges from 1.4 with 35 d.o.f. for b8 to 5.5 with 42 d.o.f. for b3). An additional component is necessary to improve the fits. We used the F-test to calculate whether the additional component was indeed significant. For the additional component we chose a black body, as is generally used when modeling the X-ray spectra of bright LMXBs (e.g. White et al. 1986; Christian \& Swank 1997; Church \& Bałucińska-Church 2001). Note that more complicated models are necessary to describe the spectra of GX $17+2$ with better energy resolution and broader energy range (e.g. Di Salvo et al. 2000). However, our approach is adequate for this paper. In the rightmost column of Table 3 we give the results of our F-tests, i.e. the probability that the addition of the black-body component in the spectral fits is not significant. We conclude 


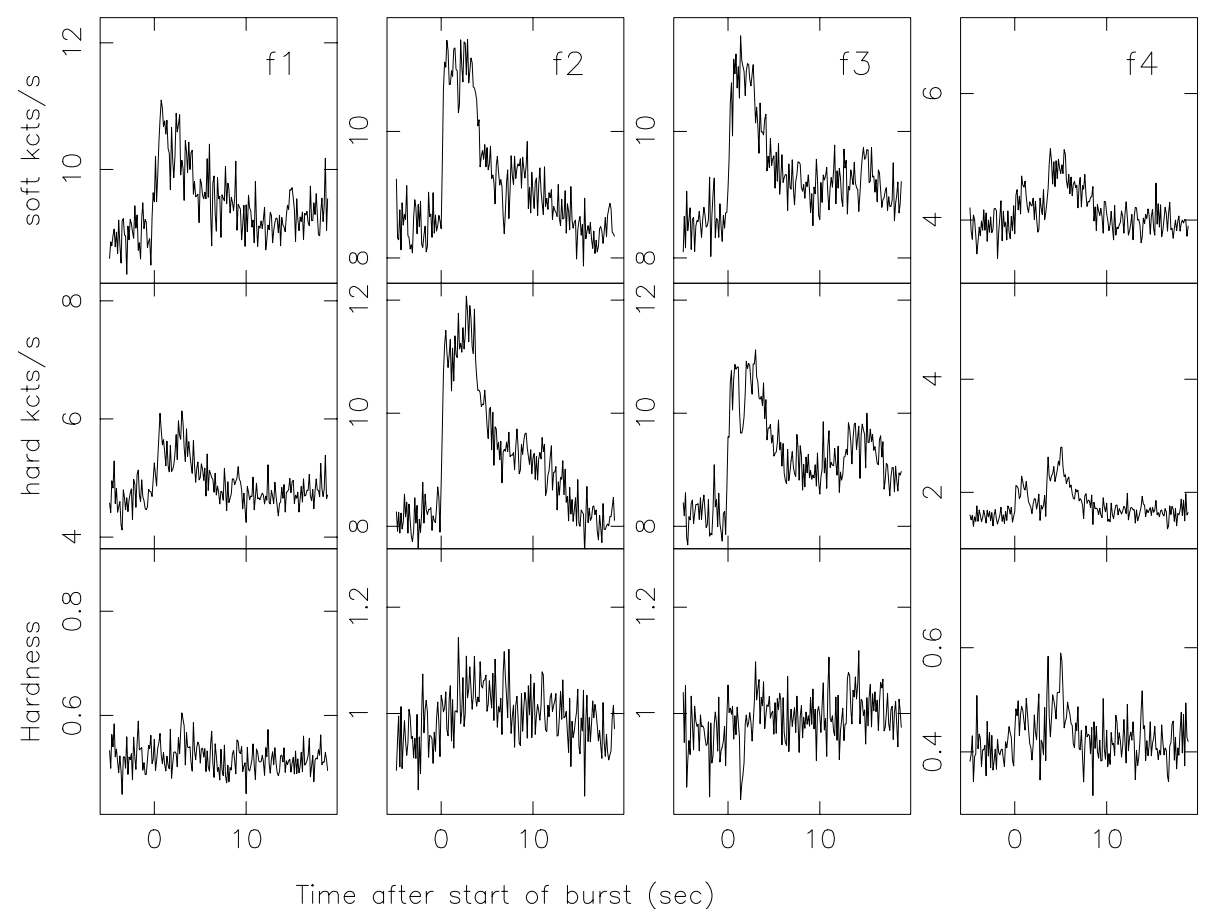

Fig. 2. The X-ray flare light curves at low (upper panels) and high (middle panels) energies and the corresponding hardness curves (lower panels), all at a time resolution of $0.125 \mathrm{~s}$, for the four flares (f1-f4; see text). Time zero is the flare start time as given in Table 2. The low and high energy ranges are $1.5-7.2 \mathrm{keV}$ and $7.2-19.7 \mathrm{keV}$, respectively, for f1, $1.9-6.2 \mathrm{keV}$ and $6.2-19.6 \mathrm{keV}$, respectively, for $\mathrm{f} 2$ and $\mathrm{f} 3$, and $2.1-7.1 \mathrm{keV}$ and $7.1-19.9 \mathrm{keV}$, respectively, for 4 . Hardness is defined as the count rate ratio of the high to low energy band. No corrections for background and dead-time have been applied.

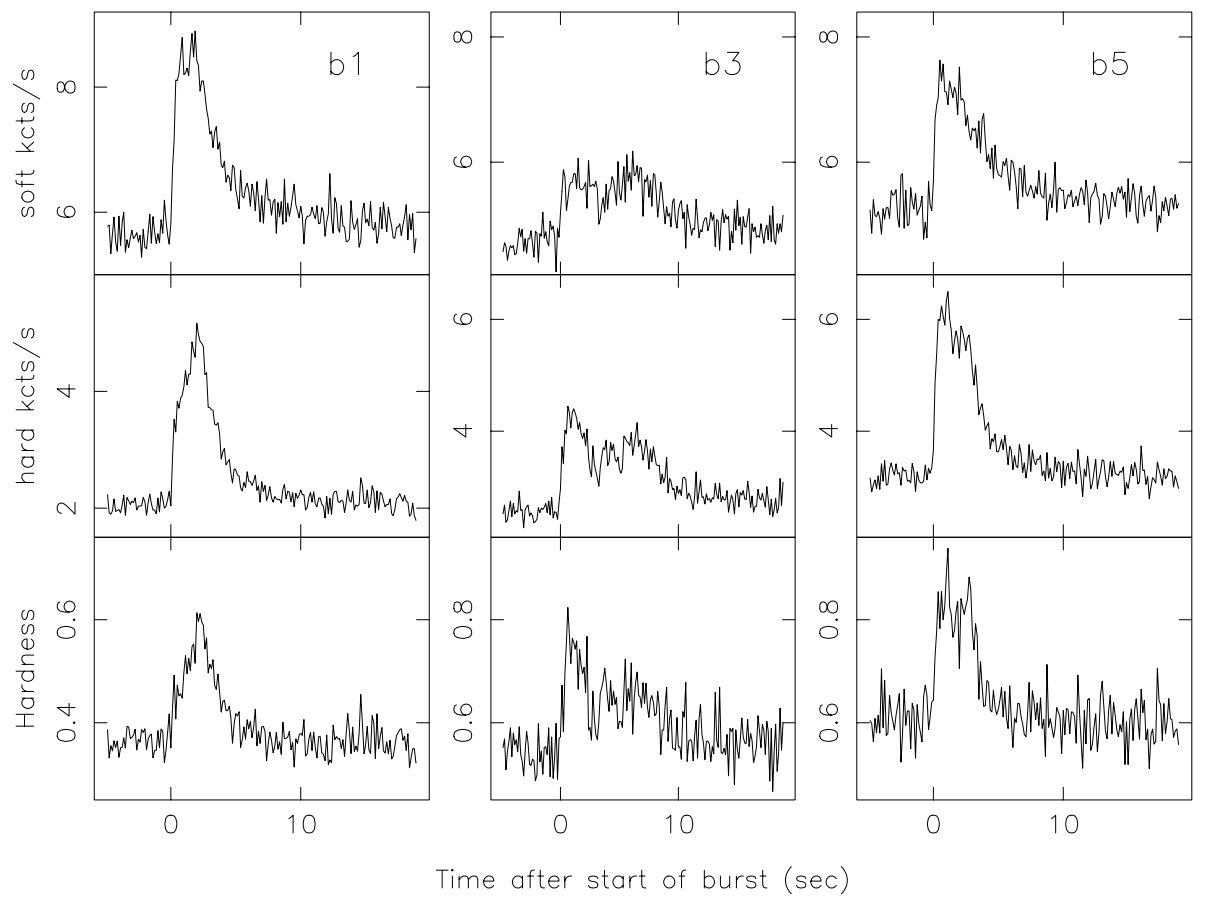

Fig. 3. Same as Fig. 2, but for the three short X-ray bursts b1, b3, and b5. The low and high energy ranges are 1-7.2 keV and 7.2-19.7 keV, respectively, for b1, whereas they are 1.9-6.2 keV and 6.2-19.6 keV, respectively, for b3 and b5.

that except for bursts b6 and b7, an additional component is warranted on the $\gtrsim 99 \%$ level. In Table 3 we present the results of the spectral fits to the persistent emission. Some of the $\chi_{\text {red }}^{2}$ values are rather small (bursts b2, b3, b5), perhaps due to a slight overestimate of the systematic error added (1\%). The unabsorbed persistent flux just before the bursts during the various observations varied between $1.9-2.5 \times 10^{-8} \mathrm{erg} \mathrm{s}^{-1} \mathrm{~cm}^{-2}(2-20 \mathrm{keV})$. For bursts b6 and b7 we determined single parameter 95\% confidence upper limits (using $\Delta \chi^{2}=2.71$ ) on the black-body 

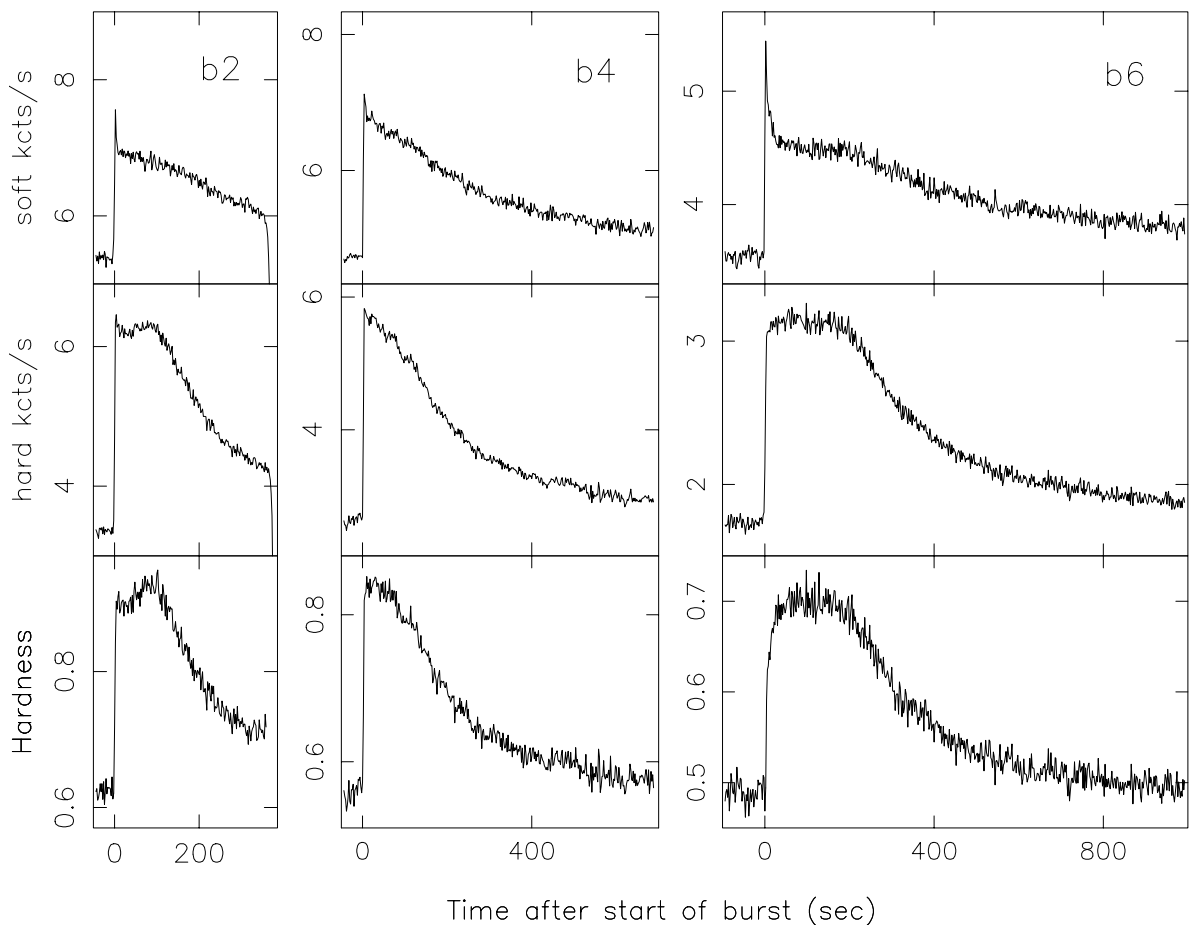

Fig. 4. Same as Fig. 3, but at a time resolution of $2 \mathrm{~s}$, for the long bursts b2, b4 and b6. Note that b2 was interrupted by a South Atlantic Anomaly (SAA) passage as can be seen by the sudden decrease in count rate. The low and high energy ranges are $1.9-6.2 \mathrm{keV}$ and $6.2-\simeq 60 \mathrm{keV}$, respectively, for b2, $1.9-6.2 \mathrm{keV}$ and $6.2-19.6 \mathrm{keV}$, respectively, for b4, and $2.1-7.1 \mathrm{keV}$ and $7.1-19.9 \mathrm{keV}$, respectively, for b6.
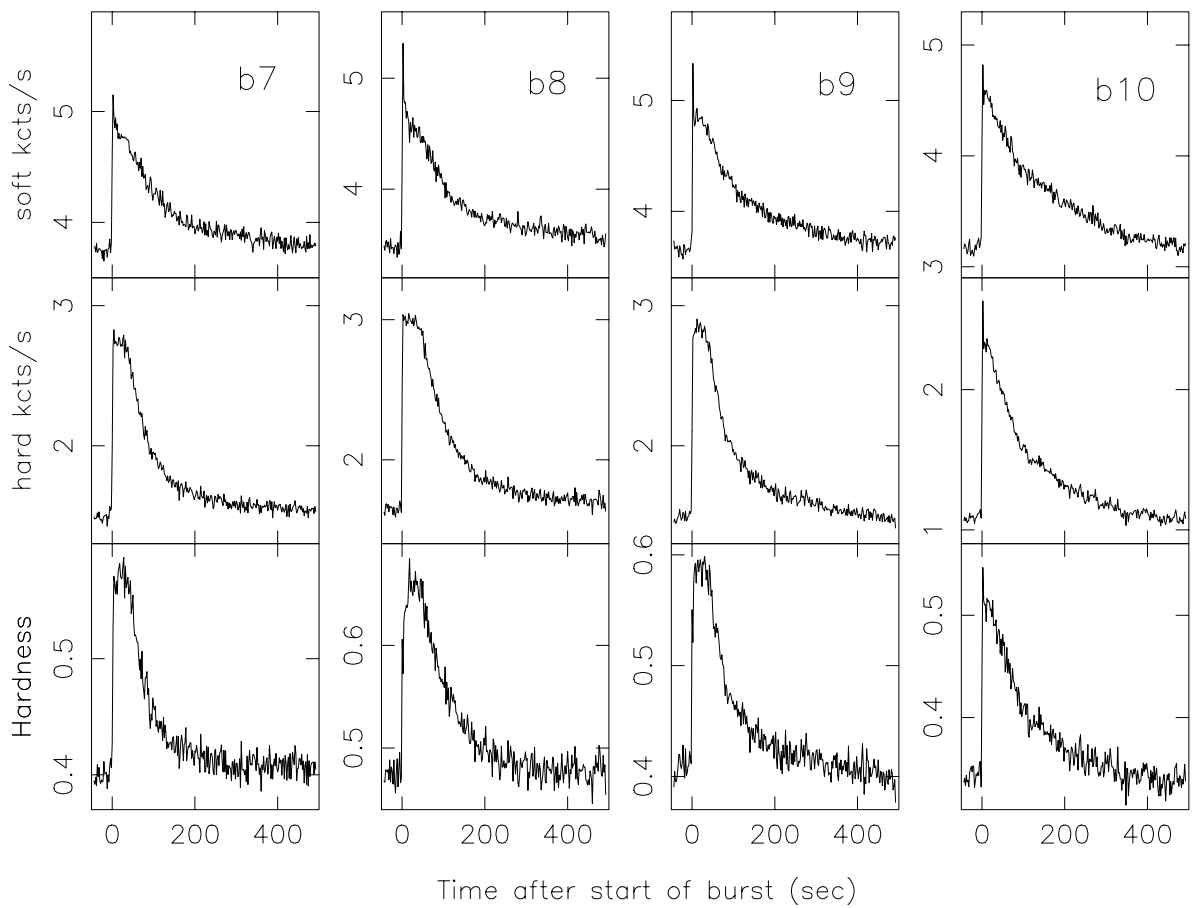

Fig. 5. Same as Fig. 4, for the last four long bursts observed in 1999 Oct. (b7-b10). The low and high energy ranges are $1.9-7.1 \mathrm{keV}$ and $7.1-19.9 \mathrm{keV}$, respectively.

contribution, by including a black-body component in the spectral fits, and fixing the temperature to its mean value derived for the persistent spectra of the other bursts, i.e. $k T_{\mathrm{bb}}=1.14 \mathrm{keV}$. The black-body component contribu- tion to the persistent emission varied between less than $2 \%$ (burst b7) up to $29 \%$ (bursts b2, b3) in the $2-20 \mathrm{keV}$ band (Table 3 ). 

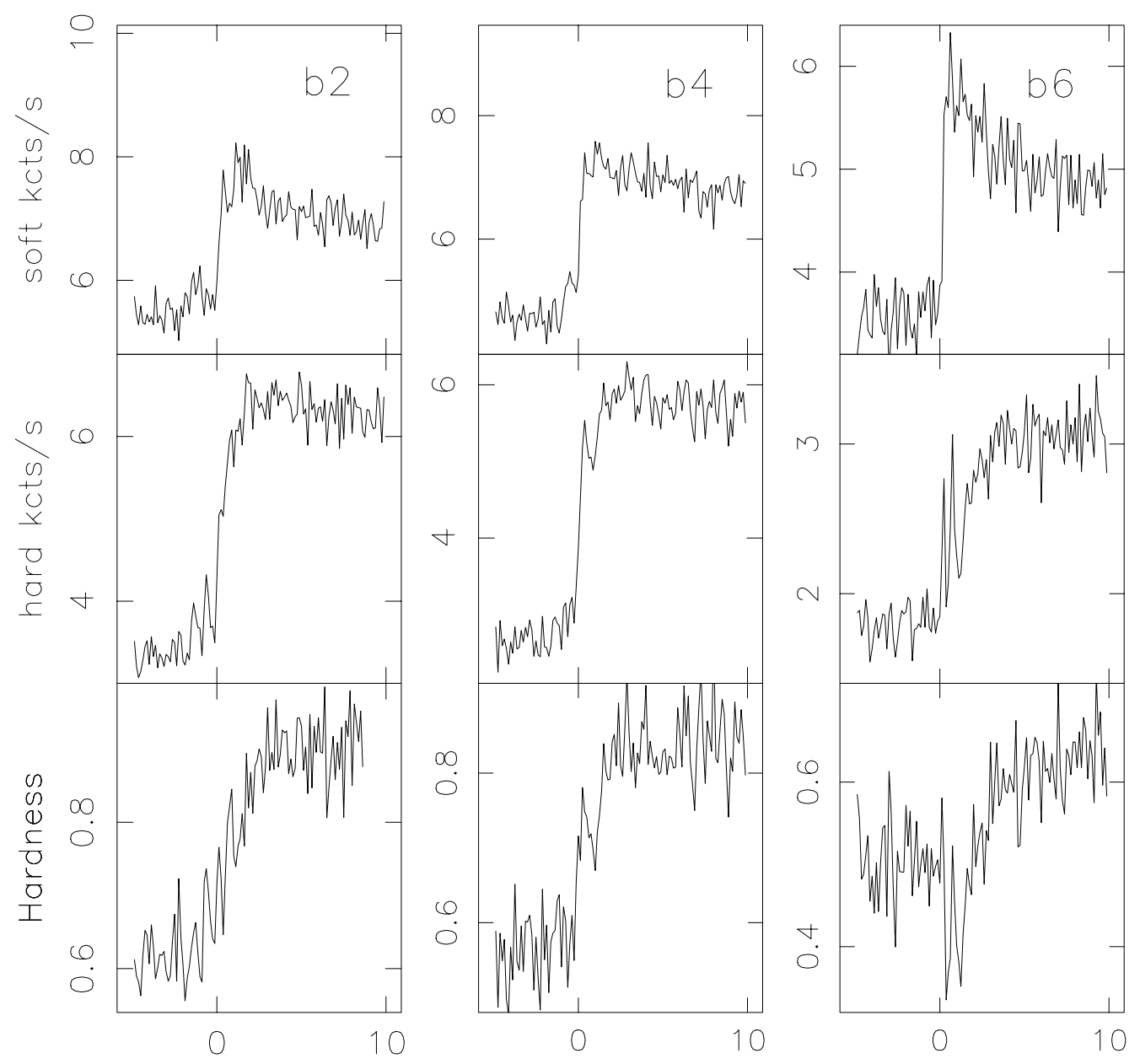

Time after start of burst (sec)

Fig. 6. Same as Fig. 4, but $5 \mathrm{~s}$ before and $10 \mathrm{~s}$ after the start of the bursts b2, b4 and b6, at a time resolution of $0.125 \mathrm{~s}$.

\subsubsection{Persistent emission during the bursts?}

\subsubsection{Previous EXOSAT results}

Usually it is assumed that the persistent emission is not influenced by the burst and that one can, therefore, study the burst by subtracting the persistent emission from the total source emission. This is referred to as the "standard" burst spectral analysis (see e.g. Sztajno et al. 1986). However, if the neutron star photosphere contributes significantly to the persistent emission, this approach is not correct, if the burst emission originates from the same region (van Paradijs \& Lewin 1986). In this case the spectral fits to the net burst spectra yield a systematically larger black-body temperature, $T_{\mathrm{bb}}$, and smaller apparent black-body radius, $R_{\mathrm{bb}}$, especially near the end of the burst when the net burst flux is low. In fact, in this case of incorrect subtraction of the persistent emission, the net burst spectrum is not a black-body. Van Paradijs \& Lewin (1986) proposed to fit the total source spectrum with a two-component model, a black-body component and a non-black-body component. During the burst the non black-body component is fixed to what is found in the persistent emission, and the black-body component is left free. The black-body component should include all emission from the neutron star photosphere. The underlying idea is that the non black-body component arises from the accretion process, and is not influenced by the X-ray burst.

GX $17+2$ is a bright X-ray source, and presumably the neutron star contributes significantly to the persistent emission (van Paradijs \& Lewin 1986; Sztajno et al. 1986). Since the source is bright, compared to most other burst sources the net flux at the peak of the burst relative to the persistent flux is rather low. Sztajno et al. (1986) found that the black-body component contributed $\simeq 40 \%$ to the persistent emission just before the two bursts observed by EXOSAT. Using the "standard" burst spectral analysis Sztajno et al. (1986) found relatively high black-body temperatures $\left(k T_{\mathrm{bb}} \simeq 2-3 \mathrm{keV}\right)$ and relatively small apparent black body radii at a distance of $10 \mathrm{kpc}\left(R_{\mathrm{bb}, 10} \simeq 3-5 \mathrm{~km}\right.$, for isotropic emission) for the short ( $10 \mathrm{~s})$ burst. For the long ( $>5 \mathrm{~min}$ ) burst, $k T_{\mathrm{bb}}$ only showed a small change from $\simeq 2.1 \mathrm{keV}$ at the peak of the burst to $\simeq 1.7 \mathrm{keV}$ near the end of the burst, with $R_{\mathrm{bb}, 10}$ decreasing from $\simeq 7 \mathrm{~km}$ to $\simeq 4 \mathrm{~km}$. Their fits were, however, satisfactory, with $\chi_{\text {red }}^{2}$ 
Table 3. Persistent emission spectral parameters ${ }^{a}$.

\begin{tabular}{|c|c|c|c|c|c|c|c|c|c|c|}
\hline$\#$ & $\begin{array}{c}F_{\text {pers }}{ }^{b} \\
(2-20 \mathrm{keV})\end{array}$ & $\begin{array}{l}k T_{\mathrm{bb}} \\
(\mathrm{keV})\end{array}$ & $\begin{array}{c}R_{\mathrm{bb}, 10}{ }^{c} \\
(\mathrm{~km})\end{array}$ & $\overline{\Gamma^{d}}$ & $\begin{array}{c}E_{\text {cut }} \\
(\mathrm{keV})\end{array}$ & norm. $^{e}$ & gnorm. ${ }^{f}$ & $\begin{array}{l}\chi_{\text {red }}^{2} / \\
\text { d.o.f. }\end{array}$ & $\mathrm{bb}^{g}$ & $P^{h}$ \\
\hline b1 & $2.0 \pm 0.3$ & $1.15 \pm 0.06$ & $14.1 \pm 1.5$ & $1.0 \pm 0.1$ & $4.5 \pm 0.2$ & $3.8 \pm 0.4$ & $0.013 \pm 0.002$ & $0.89 / 47$ & 16 & $5 \times 10^{-10}$ \\
\hline b2 & $2.2 \pm 0.5$ & $1.10 \pm 0.03$ & $22.1 \pm 2.2$ & $0.4 \pm 0.2$ & $3.8 \pm 0.2$ & $1.5 \pm 0.4$ & $0.008 \pm 0.002$ & $0.56 / 40$ & 29 & $4 \times 10^{-18}$ \\
\hline b3 & $1.9 \pm 0.3$ & $1.13 \pm 0.03$ & $19.2 \pm 1.5$ & $0.9 \pm 0.1$ & $4.2 \pm 0.2$ & $2.6 \pm 0.4$ & $0.013 \pm 0.002$ & $0.58 / 40$ & 29 & $1 \times 10^{-18}$ \\
\hline b4 & $2.0 \pm 0.3$ & $1.10 \pm 0.04$ & $20.3 \pm 1.8$ & $0.7 \pm 0.1$ & $4.0 \pm 0.2$ & $2.3 \pm 0.4$ & $0.014 \pm 0.002$ & $0.85 / 40$ & 28 & $6 \times 10^{-15}$ \\
\hline b5 & $2.3 \pm 0.3$ & $1.16 \pm 0.05$ & $17.5 \pm 1.7$ & $0.9 \pm 0.1$ & $4.5 \pm 0.2$ & $3.2 \pm 0.4$ & $0.010 \pm 0.002$ & $0.59 / 40$ & 23 & $5 \times 10^{-15}$ \\
\hline b6 & $2.5 \pm 0.1$ & - & - & $1.03 \pm 0.03$ & $5.1 \pm 0.1$ & $4.8 \pm 0.1$ & $0.012 \pm 0.002$ & $0.71 / 35$ & $<7^{i}$ & 8 \\
\hline b7 & $2.5 \pm 0.1$ & - & - & $1.17 \pm 0.03$ & $4.6 \pm 0.1$ & $7.0 \pm 0.2$ & $0.015 \pm 0.002$ & $1.22 / 35$ & $<2^{i}$ & 30 \\
\hline b8 & $2.4 \pm 0.5$ & $1.08 \pm 0.13$ & $11.0 \pm 4.3$ & $1.0 \pm 0.1$ & $5.3 \pm 0.3$ & $3.8 \pm 0.5$ & $0.011 \pm 0.002$ & $1.13 / 33$ & 6 & 1 \\
\hline b9 & $2.4 \pm 0.4$ & $1.26 \pm 0.08$ & $11.6 \pm 1.5$ & $1.0 \pm 0.1$ & $4.9 \pm 0.3$ & $4.3 \pm 0.4$ & $0.010 \pm 0.003$ & $0.87 / 33$ & 13 & $8 \times 10^{-5}$ \\
\hline b10 & $1.9 \pm 0.3$ & $1.18 \pm 0.05$ & $15.1 \pm 1.4$ & $1.1 \pm 0.1$ & $4.5 \pm 0.3$ & $3.7 \pm 0.5$ & $0.011 \pm 0.002$ & $1.16 / 33$ & 22 & $5 \times 10^{-7}$ \\
\hline
\end{tabular}

${ }^{a} N_{\mathrm{H}}$ was fixed to $2 \times 10^{22}$ atoms $\mathrm{cm}^{-2}$, see text.

${ }^{b}$ Unabsorbed persistent flux in $10^{-8} \mathrm{erg} \mathrm{s}^{-1} \mathrm{~cm}^{-2}$.

${ }^{c}$ Apparent black-body radius at $10 \mathrm{kpc}$.

${ }^{d}$ Power-law photon index.

${ }^{e}$ Power-law normalization in photons $\mathrm{keV}^{-1} \mathrm{~cm}^{-2} \mathrm{~s}^{-1}$ at $1 \mathrm{keV}$.

${ }^{f}$ Normalization in photons $\mathrm{cm}^{-2} \mathrm{~s}^{-1}$ in the line modeled by a Gaussian fixed at $6.7 \mathrm{keV}$ and width $(\sigma)$ of $0.1 \mathrm{keV}$.

${ }^{g}$ Black-body emission contribution to the total persistent flux in \% (2-20 keV).

${ }^{h}$ Probability (in \%) that inclusion of a black-body component is not significant.

${ }^{i} 95 \%$ confidence upper limit, using $k T_{\mathrm{bb}}=1.14 \mathrm{keV}$, see text.
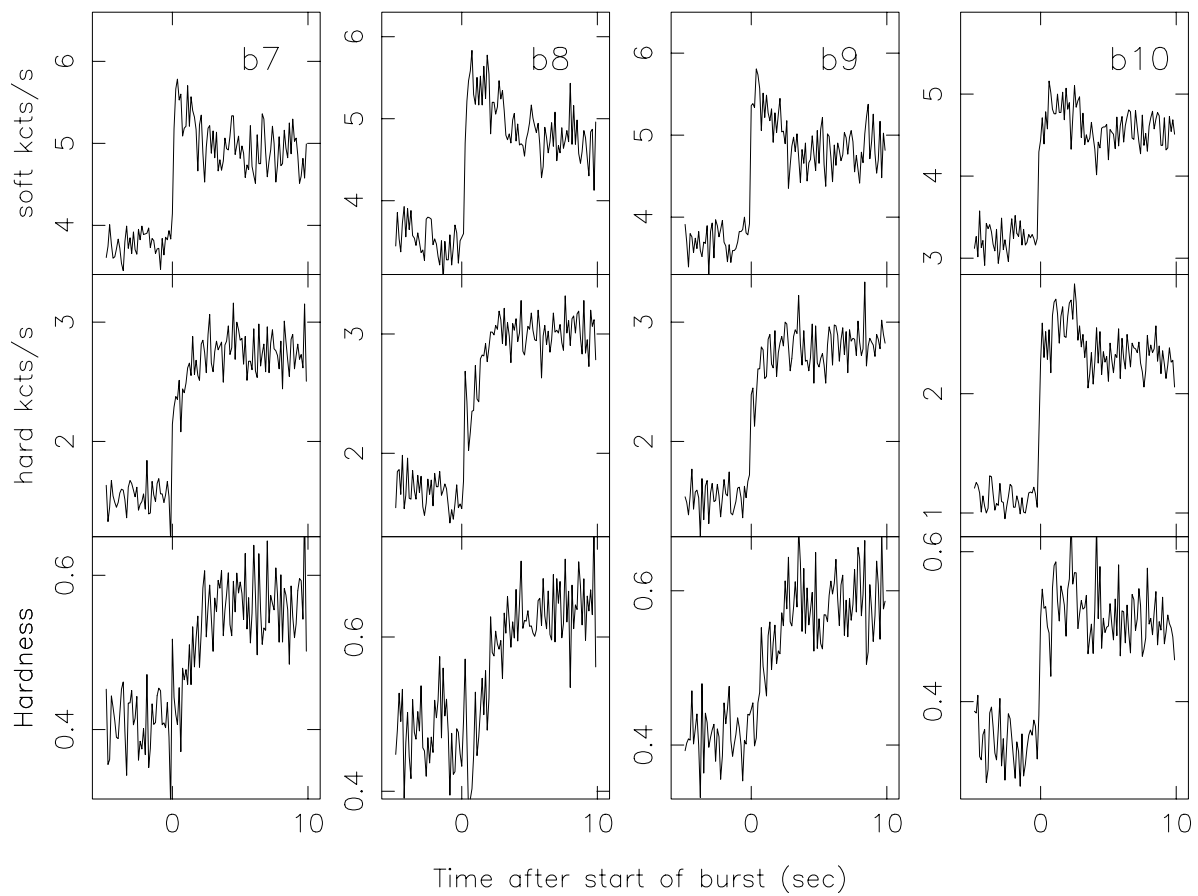

Fig. 7. Same as Fig. 5, but $5 \mathrm{~s}$ before and $10 \mathrm{~s}$ after the start of the last four bursts observed in 1999 Oct. (b7-b10), at a time resolution of $0.125 \mathrm{~s}$.

of 0.6-1.2. Using the two-component model, Sztajno et al. (1986) found that during the short burst the values for $T_{\mathrm{bb}}$ were in the range normally seen in type I X-ray bursts, and that the systematic decrease in $R_{\mathrm{bb}}$ had disappeared. The $\chi_{\text {red }}^{2}$ values for the two-component model fits ranged between 0.6 and 1.3, except for one fit, for which $\chi_{\text {red }}^{2}=1.6$, i.e. slightly worse than the "standard" spectral analysis. They attributed this to the smaller relative error in the total burst data than in the net-burst data, for which the persistent emission was subtracted. Note that in this case Sztajno et al. (1986) found a slight increase in $R_{\mathrm{bb}, 10}$, apparently anti-correlated with $T_{\mathrm{bb}}$, which they 
argue was due to the non-Planckian shape of the spectrum of a hot neutron star (van Paradijs 1982; see Titarchuk 1994; Madej 1997, and references therein; see, however, Sect. 5.2).

\subsubsection{Our RXTE results}

Guided by the results of Sztajno et al. (1986) discussed in Sect. 3.2.2.1, we first fitted the total burst data using a black body and a cut-off power law plus a Gaussian line. The parameters of the absorbed cut-off power law and Gaussian line were fixed to the values found for the persistent emission before the burst (Table 3). Using this model we obtained good fits to the $16 \mathrm{~s}$ spectra of bursts b6 and b7, for which the persistent emission did not contain a significant black-body contribution $\left(\chi_{\text {red }}^{2}\right.$ of $\simeq 1$ for 37 d.o.f.). However, the 16 s spectral fits were bad whenever the persistent emission spectra contained a blackbody component; the fits became worse as the persistent black-body contribution became stronger (for the first $\sim 100$ s of the burst: $\chi_{\text {red }}^{2} /$ d.o.f. $\simeq 1.5-3 / 37$ [burst b8], $\chi_{\text {red }}^{2} /$ d.o.f. $\simeq 2.5-8 / 37\left[\right.$ burst b9], $\chi_{\text {red }}^{2} /$ d.o.f. $\simeq 5-10 / 37$ [burst b10], $\chi_{\text {red }}^{2} /$ d.o.f. $\simeq 20-24 / 44$ [burst b4], $\chi_{\text {red }}^{2} /$ d.o.f. $\simeq 22-25 / 44$ [burst b2]). The worst $\chi_{\text {red }}^{2}$ occurred near the peak of the bursts. For instance, Fig. 8 shows the best-fit parameters and $\chi_{\text {red }}^{2}$ for burst b4, for which the persistent emission had a black-body contribution of $\simeq 28 \%(2-$ $20 \mathrm{keV})$. An example of a burst spectrum and the result of the two-component fit is shown in Fig. 9a for burst b2. The $\chi_{\text {red }}^{2}$ with 44 d.o.f. for burst b4 decreases from $\simeq 24$ at the beginning of the burst to $\simeq 2$ near the end of the burst. A similar trend is seen for the $0.25 \mathrm{~s}$ burst spectral fits: $\chi_{\text {red }}^{2}$ of $\simeq 2$ for 18 d.o.f. during the first $\simeq 10$ s of burst b4 and an $\chi_{\text {red }}^{2}$ of $\simeq 1$ for 16 d.o.f. during the first $\simeq 10$ s of burst b6 (see Fig. 8). The $\chi_{\text {red }}^{2}$ values for $0.25 \mathrm{~s}$ spectral fits were much lower than those for the $16 \mathrm{~s}$ spectral fits, due to the much lower signal to noise and lower spectral resolution of the $0.25 \mathrm{~s}$ spectra. Note that the high values of $\chi_{\text {red }}^{2}$ for the $16 \mathrm{~s}$ spectra are not due to fast spectral variations within the time the spectra are accumulated. This only applies to spectra which include the first few seconds of the bursts, during the short radius expansion and initial contraction phase (see Sect. 3.2.4). Halfway the decline of burst b4, $R_{\mathrm{bb}}$ seems to increase again; this is also seen in the other long bursts (except burst b6), and in the short bursts (e.g. burst b1, see Fig. 8). This is similar to that seen by Sztajno et al. (1986), but their fits seemed better (see Sect. 3.2.2). This is (probably) due to the much lower signal to noise ratio of the EXOSAT/ME data.

The mean bolometric black-body flux during the

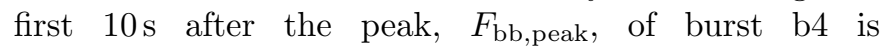
higher than that of burst b6. The difference is $0.63( \pm 0.12) \times 10^{-8} \mathrm{erg} \mathrm{s}^{-1} \mathrm{~cm}^{-2}$ (for the uncertainty in $F_{\text {bb,peak }}$ we used the rms variation in the bolometric black-body fluxes). This is consistent with the bolometric black body contribution in the persistent emission, $F_{\text {bb,pers, }}$, before burst b4 (indicated by the dotted lines in the top panels for burst b4 in Fig. 8): $F_{\text {bb,pers }}=$ $0.65( \pm 0.05) \times 10^{-8} \mathrm{erg} \mathrm{s}^{-1} \mathrm{~cm}^{-2}$. We measured $F_{\mathrm{bb}, \text { peak }}$ and $F_{\mathrm{bb} \text {,pers }}$ for all the bursts to see if this effect is seen in other bursts as well. We used the highest observed black body flux, except for burst b2 where we used the first $16 \mathrm{~s}$ measurement after the start of the burst. The result is displayed in Fig. 10a. Indeed, $F_{\text {bb,peak }}$ differs between the bursts and is clearly correlated with $F_{\mathrm{bb} \text {,pers }}$ (except for burst b3). Such a relation can be expected if $F_{\text {bb,peak }}$ is close to a certain upper limit (presumably the Eddington limit) and includes a (certain fraction) of the persistent emission. We can reasonably fit the data points to the function $F_{\mathrm{bb} \text {,peak }}=F_{\mathrm{bb} \text {,pers }}+C$ (excluding bursts b2 and b3; burst b2 did not cover the first $10 \mathrm{~s}$ after the peak of the burst, and burst b3 was weaker than the other bursts). The resulting $\chi_{\text {red }}^{2}=2.0$ for 7 d.o.f. We find $C=(1.31 \pm 0.03) \times 10^{-8} \mathrm{erg} \mathrm{s}^{-1} \mathrm{~cm}^{-2}$; this is close to the values of $F_{\mathrm{bb} \text {,peak }}$ for bursts b6 and b7 which had no significant black-body contribution in the preceding persistent emission. In Fig. 10 we have also indicated whether a burst was a radius expansion event (see Sect. 3.2.4). It can be seen in Fig. 10a that the bursts with no radius expansion/contraction phase (except burst b3) have somewhat higher values of $F_{\mathrm{bb}}$,peak than the radius expansion bursts (except burst b4). Theoretically, during the radius expansion/contraction phases the flux should equal the Eddington limit. Therefore, the (peak) fluxes observed for different radius expansion bursts should have similar values, whereas for bursts with no radius expansion/contraction phase they should be either similar or smaller. This is not the case.

To summarize, we find that the method proposed by van Paradijs \& Lewin (1986) and applied by Sztajno et al. (1986) to the bursts observed by EXOSAT does not work for our bursts observed with the RXTE/PCA. The twocomponent spectral fits during the bursts give bad results whenever there is a black-body contribution to the perburst persistent emission. Moreover, the total burst peak fluxes, $F_{\text {bb,peak }}$ are different from burst to burst and depend on the amount of the black-body contribution to the persistent emission, $F_{\mathrm{bb} \text {,pers }}$. This is not what one would expect if during some of the bursts a limit is reached (presumably the Eddington limit). We conclude that the persistent black-body emission does not disappear during the burst. An example is displayed in Fig. 9b. We show again the first $16 \mathrm{~s}$ spectrum during burst b2 plus now the results of a three-component fit, i.e. two black bodies and a cutoff power-law plus Gaussian line (subjected to interstellar absorption). The parameters of the cut-off power law plus Gaussian line and one black-body component have been fixed to those found for the persistent emission (so the fit does have the same number of d.o.f. as the one displayed in Fig. 9a). Clearly, the fit has improved considerably. This means that the burst emission is decoupled from the persistent emission, and therefore the "standard" spectral analysis should work in this case. This is the subject of the next subsection. 


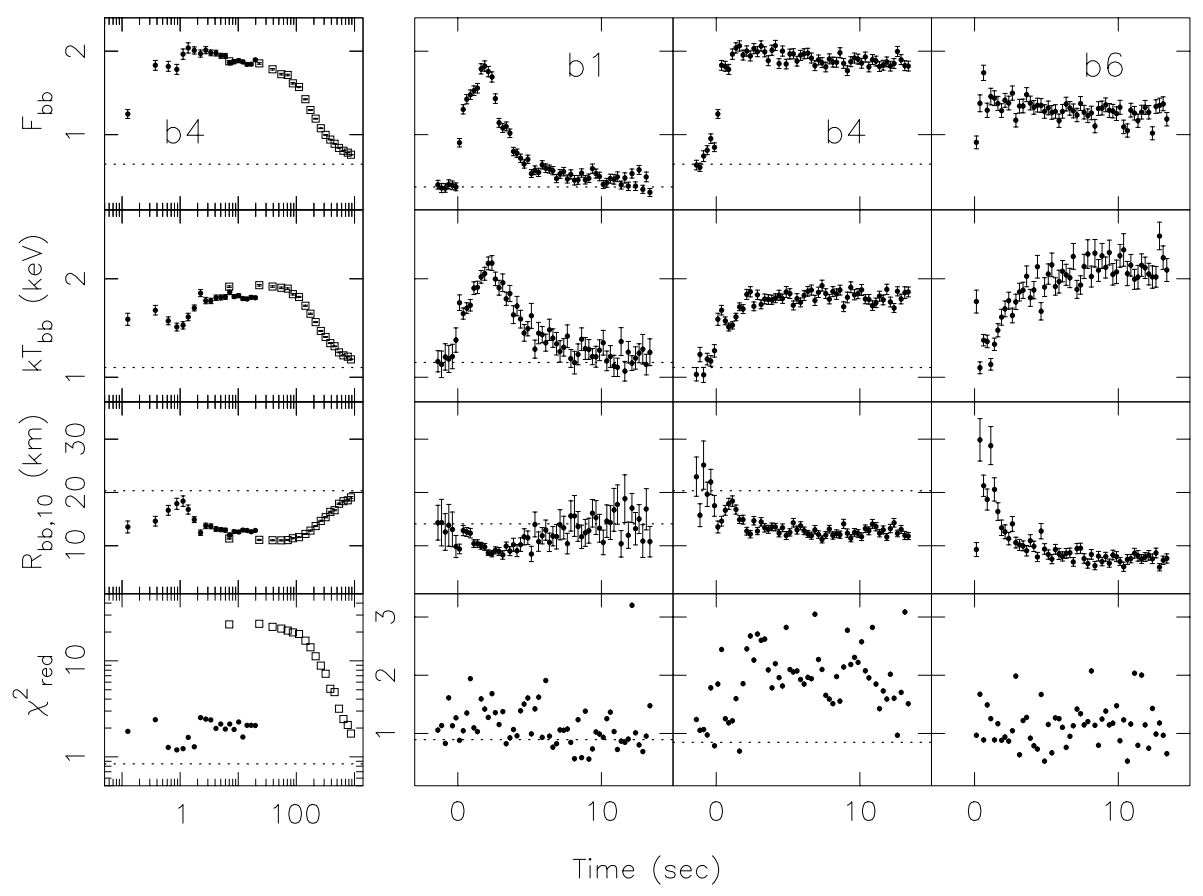

Fig. 8. Leftmost panel: two-component spectral fit results for the total burst emission of burst b4 plotted on a logarithmic time scale. The filled circles and open squares represent the fit results of the $0.25 \mathrm{~s}$ and $16 \mathrm{~s}$ spectra, respectively. The data have been logarithmically rebinned in time for clarity. The values for the persistent black-body component have been indicated by a dotted line. Right panels: two-component spectral fit results for the $0.25 \mathrm{~s}$ spectra of the total burst emission of burst b1, b4 and b6, see text. The values for the persistent black-body component have been indicated by a dotted line for burst b1 and b4. Both panels: from top to bottom: bolometric black-body flux, $F_{\mathrm{bb}}$, in $10^{-8} \mathrm{erg} \mathrm{s}^{-1} \mathrm{~cm}^{-2}$, black-body temperature, $k T_{\mathrm{bb}}$, apparent black-body radius, $R_{\mathrm{bb}, 10}$, at $10 \mathrm{kpc}$, and goodness of fit expressed in $\chi_{\mathrm{red}}^{2}$. For bursts b1, b4 and b6 the number of d.o.f. is 22,18 and 16 , respectively, for the $0.25 \mathrm{~s}$ spectral fits. For burst b4 the number of d.o.f. is 44 for the $16 \mathrm{~s}$ spectral fits. Note the difference in scales of $\chi_{\text {red }}^{2}$ in the leftmost panel with respect to the other panels.
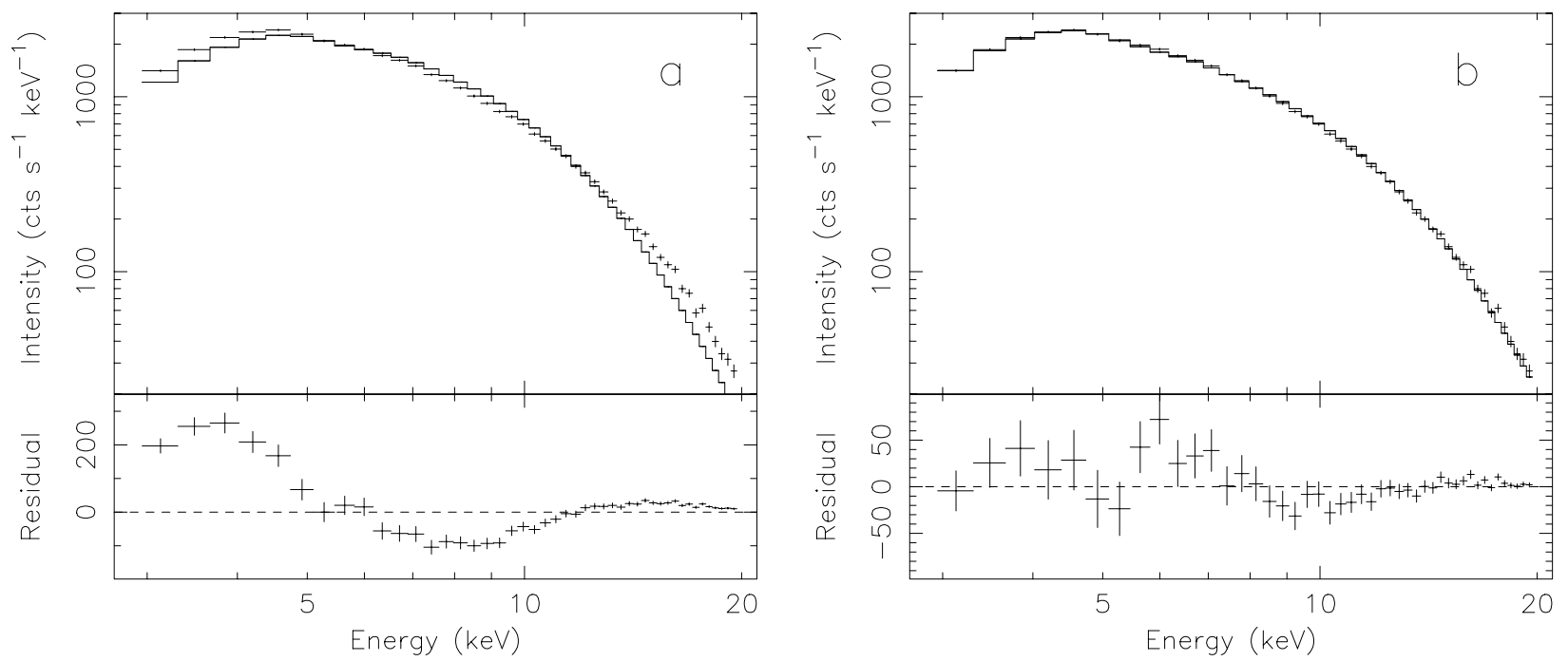

Fig. 9. a) At the top the first $16 \mathrm{~s}$ spectrum observed after the start of burst b2 is displayed. A two-component fit is shown, i.e. a single black-body and cut-off power-law plus Gaussian line (subjected to interstellar absorption). The parameters of the cut-off power-law component plus Gaussian line are fixed to the values derived for the persistent emission. At the bottom the residuals after subtracting the best model from the observed spectrum is displayed. The fit is clearly bad $\left(\chi_{\text {red }}^{2} /\right.$ d.o.f. $\left.=24.2 / 44\right)$. b) At the top the same first $16 \mathrm{~s}$ spectrum at the beginning of burst b2 is displayed. Now a three-component fit is shown, i.e. two black-body components and one cut-off power-law component (subjected to interstellar absorption). The cut-off power-law and one black-body component parameters are fixed to the values derived for the persistent emission. At the bottom the residuals after subtracting the best model from the observed spectrum is displayed. The fit has clearly improved $\left(\chi_{\text {red }}^{2} /\right.$ d.o.f. $\left.=1.8 / 44\right)$. 


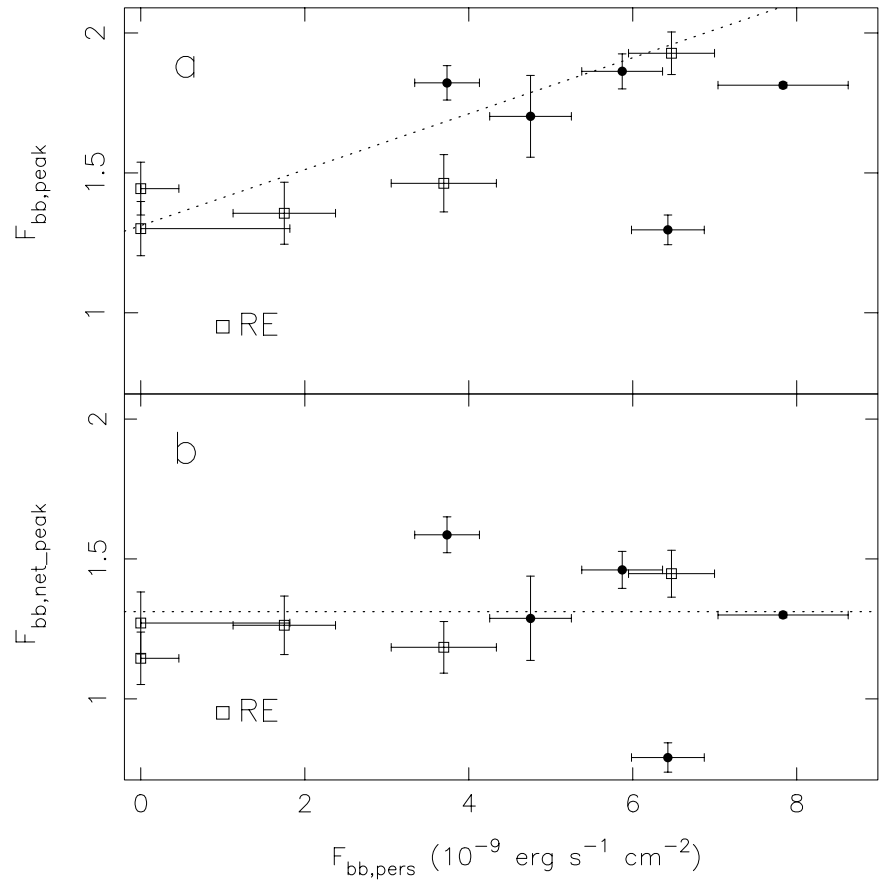

Fig. 10. a) Bolometric peak black-body flux (see text), $F_{\text {bb,peak }}$, in units of $10^{-8} \mathrm{erg} \mathrm{s}^{-1} \mathrm{~cm}^{-2}$ versus the bolometric persistent black-body flux, $F_{\mathrm{bb} \text {,pers }}$. Radius expansion (RE) bursts are denoted with an open square. The dotted line represents a fit to the data points (excluding burst b2 and b3) to the function $F_{\mathrm{bb} \text {,peak }}=F_{\mathrm{bb} \text {,pers }}+C$. The constant $C$ corresponds to $(1.31 \pm 0.03) \times 10^{-8} \mathrm{erg} \mathrm{s}^{-1} \mathrm{~cm}^{-2}$. b) Bolometric peak black body flux from spectral fits after subtraction of the persistent emission (see text), $F_{\mathrm{bb}, \text { net_peak }}$, in units of $10^{-8} \mathrm{erg} \mathrm{s}^{-1} \mathrm{~cm}^{-2}$ versus $F_{\mathrm{bb} \text {,pers }}$. Radius expansion (RE) bursts are denoted with an open square. The dotted line represents the constant $C$.

\subsection{3. "Standard" spectral analysis: Net burst emission}

When modeling the net burst spectra (i.e. spectra obtained after subtraction of the pre-burst persistent emission from the total source spectrum) by a black body, the spectral fits are almost all satisfactory $\left(\chi_{\text {red }}^{2}\right.$ values of $\simeq 1$ ). The fit results are shown for the 3 short bursts in Fig. 11 (b1, b3, b5) and for the 7 long bursts in Fig. 12 (b2, b4, b7-b10) and 13 (left panel; b6). For the long bursts we used a logarithmic time scale, to emphasize the start of the bursts, where changes in the parameters are most rapid.

The fits are, while much improved overall, still not optimal during the peaks of the long bursts b4, b6 and $\mathrm{b} 10\left(\chi_{\text {red }}^{2}=2-3\right.$ with $44[\mathrm{~b} 4]$ or 37 [b6,b10] d.o.f. for the spectral fits to the $16 \mathrm{~s}$ spectra). In Fig. 14 we show the average net burst spectrum during the flat top part of burst b6 excluding the radius expansion and initial contraction phase (Sect. 3.2.4), i.e. $27-187 \mathrm{~s}$ after the start of the burst. $T_{\mathrm{bb}}$ and $R_{\mathrm{bb}, 10}$ do not change much during this interval. Clearly, deviations occur below $\simeq 10 \mathrm{keV}$; the intensity drops below $\simeq 5.5 \mathrm{keV}$, while there is an excess between $\simeq 5.5-8 \mathrm{keV}$. These deviations are much larger than the calibration uncertainties.

In the short bursts $T_{\mathrm{bb}}$ decreases during the decay, indicating cooling of the neutron star, as already noted from the hardness curves. There are some slight variations in $R_{\mathrm{bb}, 10}$ and $T_{\mathrm{bb}}$ during the first few seconds. However, considering the behaviour of the net burst black-body flux, $F_{\text {bb,net }}$, they do not show the correlations that would be expected for radius expansion events, i.e. an increase in $R_{\mathrm{bb}, 10}$ with a simultaneous drop in $T_{\mathrm{bb}}$ and a (nearly) constant $F_{\mathrm{bb}, \text { net }}$.

The long bursts all show more or less the same behaviour (note again that for burst b2 we have no short time scale spectral information, see Fig. 12). After a fast increase $(\lesssim 0.5 \mathrm{~s})$ to maximum, $F_{\mathrm{bb} \text {,net }}$ remains constant $\left(1.4-1.7 \times 10^{-8} \mathrm{erg} \mathrm{s}^{-1} \mathrm{~cm}^{-2}\right.$ for the various bursts) for 50 $200 \mathrm{~s}$, and then decays exponentially. The duration of this flat-topped phase is similar to that seen in the light curve at high energies (see middle panels of Figs. 4 and 5). During the first part $T_{\mathrm{bb}}$ increases, then it levels off, to decrease again when $F_{\mathrm{bb} \text {,net }}$ decreases.

$R_{\mathrm{bb}, 10}$ is $\simeq 5 \mathrm{~km}$ during the decay of the burst, until $F_{\text {bb,net }}$ drops below typically $0.2 \times 10^{-8} \mathrm{erg} \mathrm{s}^{-1} \mathrm{~cm}^{-2}$. Then $R_{\mathrm{bb}, 10}$ decreases again. Note that this latter behaviour resembles the systematic effects described by van Paradijs \& Lewin (1986) near the end of the burst, when the persistent emission becomes important with respect to the net burst emission (Sect. 3.2.2.1). However, we have argued above that the burst black-body emission is decoupled from the persistent black-body emission. It is unlikely that this behaviour is due to the fact that we assumed a constant persistent component during the bursts, while in fact the persistent component may vary on the same time scale of the burst or faster (see Sect. 3.1). If that was the case, one would expect that in some bursts $R_{\mathrm{bb}}$ would decrease, whereas in some others it would increase. On the contrary, we find that $R_{\mathrm{bb}}$ decreases in all bursts. We will discuss this issue in Sect. 5.2.2.

\subsubsection{Radius expansion bursts}

The most dramatic changes in the spectral parameters can be seen during the first few seconds of the long bursts: while there is a short dip in $T_{\mathrm{bb}}$ near the start of the burst, $R_{\mathrm{bb}, 10}$ shows values which are up to a factor of $\simeq 6$ larger than those found later on (although this is not true for burst b10, see Fig. 12). This is typical of radius expansion/contraction episodes, as described in Sect. 1. Burst b6 shows this behaviour clearest, and may have had even two of such episodes within one second (Fig. 13, left panel). The expansion to maximum radius takes place in less than $\simeq 0.5 \mathrm{~s}$, while the initial contraction phases are longer: it takes between 5 and $20 \mathrm{~s}$ from maximum expansion back to close to the neutron star surface. The final contraction phase lasts between $\simeq 20$ and $\simeq 180$ s We conclude that bursts $\mathrm{b} 4$, and $\mathrm{b} 6-\mathrm{b} 9$ are radius expansion bursts.

In Fig. 10b we show the (average) net-burst peak fluxes, $F_{\mathrm{bb}, \text { net_peak }}$, determined in the same way as in the previous subsection for $F_{\mathrm{bb} \text {,peak }}$, but now for the netburst emission. Most of the net burst peak fluxes are in the same range. For the radius expansion bursts this is 


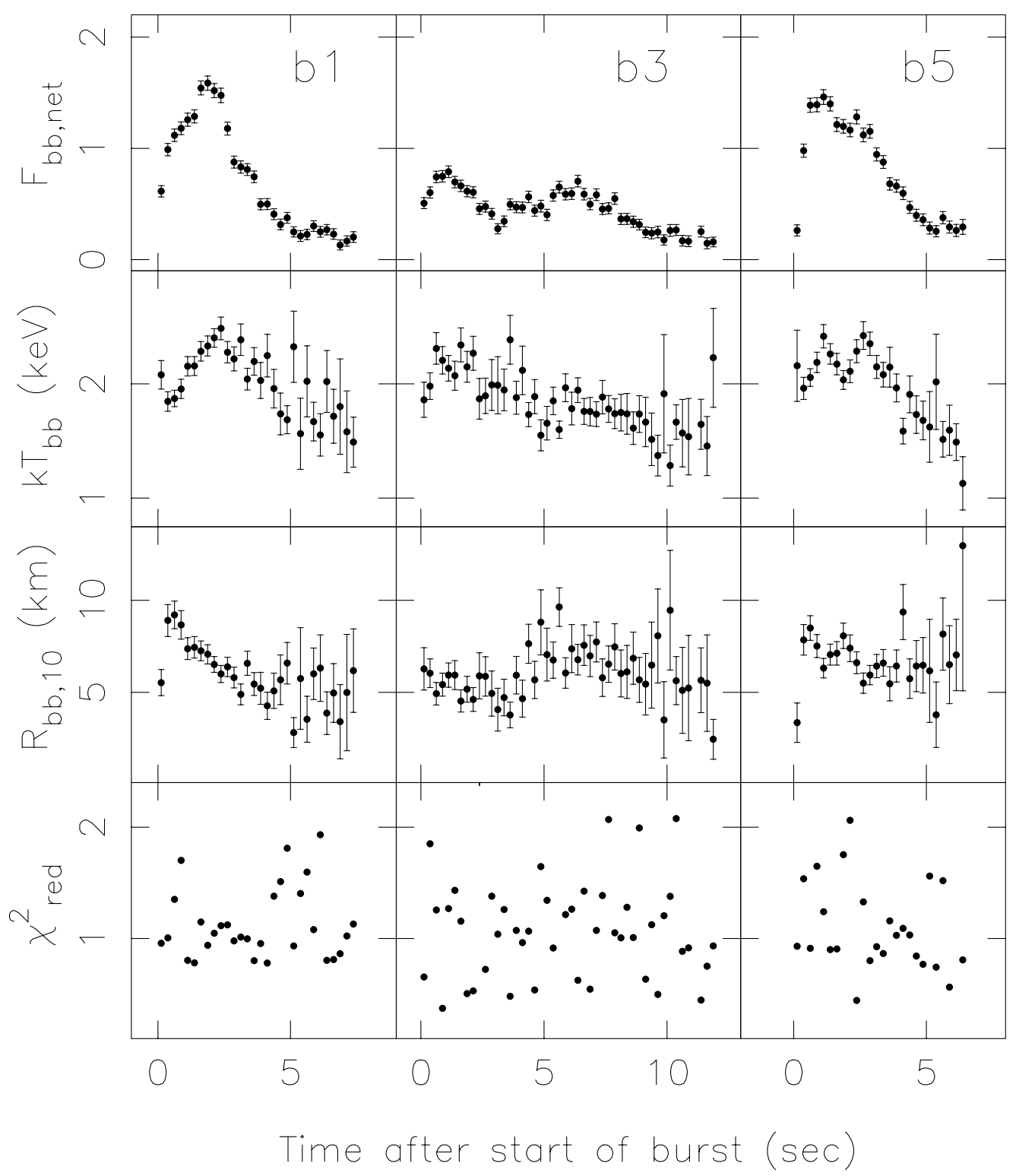

Fig. 11. Spectral fit results for the net burst emission of burst b1, b3 and b5; from top to bottom: bolometric black-body flux, $F_{\mathrm{bb}, \text { net }}$, in $10^{-8} \mathrm{erg} \mathrm{s}^{-1} \mathrm{~cm}^{-2}$, black-body temperature, $k T_{\mathrm{bb}}$, apparent black-body radius, $R_{\mathrm{bb}, 10}$, at $10 \mathrm{kpc}$, and goodness of fit expressed in reduced $\chi^{2}$. The number of d.o.f. is 22 for burst b1 and 18 for bursts b3 and b5.

expected, if the Eddington limit is reached. Note that $F_{\text {bb,peak }} \simeq F_{\text {bb,net_peak }}$ for bursts b6 and b7, as expected if there is no black-body contribution to the persistent emission. We conclude that $F_{\mathrm{bb} \text {,net_peak }}$ in the radius expansion bursts is a measure of the Eddington luminosity observed at Earth. We find that $F_{\mathrm{bb} \text {,net_peak }}$ for the non-radius expansion bursts (except bursts b3) is more or less similar to the maximum flux for the radius expansion bursts.

Radius expansion events are most conveniently displayed in a flux-temperature diagram (where temperature increases from right to left, analogous to HRdiagrams). Our best example, burst b6, is displayed this way in the right-hand panel of Fig. 13. In this diagram the radius expansion/contraction phase and the subsequent cooling of the neutron star are clearly distinguished by two separate tracks (see e.g. Lewin et al. 1993). The data points distributed along the horizontal line in the upper part of the diagram (i.e. nearly constant $F_{\mathrm{bb}, \text { net }}$ of $1.2-1.3 \times 10^{-8} \mathrm{erg} \mathrm{s}^{-1} \mathrm{~cm}^{-2}$ ) represent the expansion/contraction phase. The data points distributed along the diagonal line from the upper left to the lower right part of the diagram are from the cooling phase of the burst. We note that the point in between the two tracks is from the very first rise phase. Comparing both panels of Fig. 13 it is apparent that the burst spends a long time near the vertex of both tracks, about $150 \mathrm{~s}$, where $F_{\mathrm{bb}, \text { net }} \simeq 1.24 \times 10^{-8} \mathrm{erg} \mathrm{s}^{-1} \mathrm{~cm}^{-2}$ and $k T_{\mathrm{bb}} \simeq 2.65 \mathrm{keV}$. The first part $\left(F_{\mathrm{bb}, \text { net }} \gtrsim 0.4 \times 10^{-8} \mathrm{erg} \mathrm{s}^{-1} \mathrm{~cm}^{-2}\right)$ of the cooling track is well described ( $\chi_{\text {red }}^{2}=0.6$ for 9 d.o.f.) by a straight line with a slope of $4.14 \pm 0.15$ (dotted line in Fig. 13$)$, i.e. $F_{\mathrm{bb}, n e t}$ is consistent with being proportional 


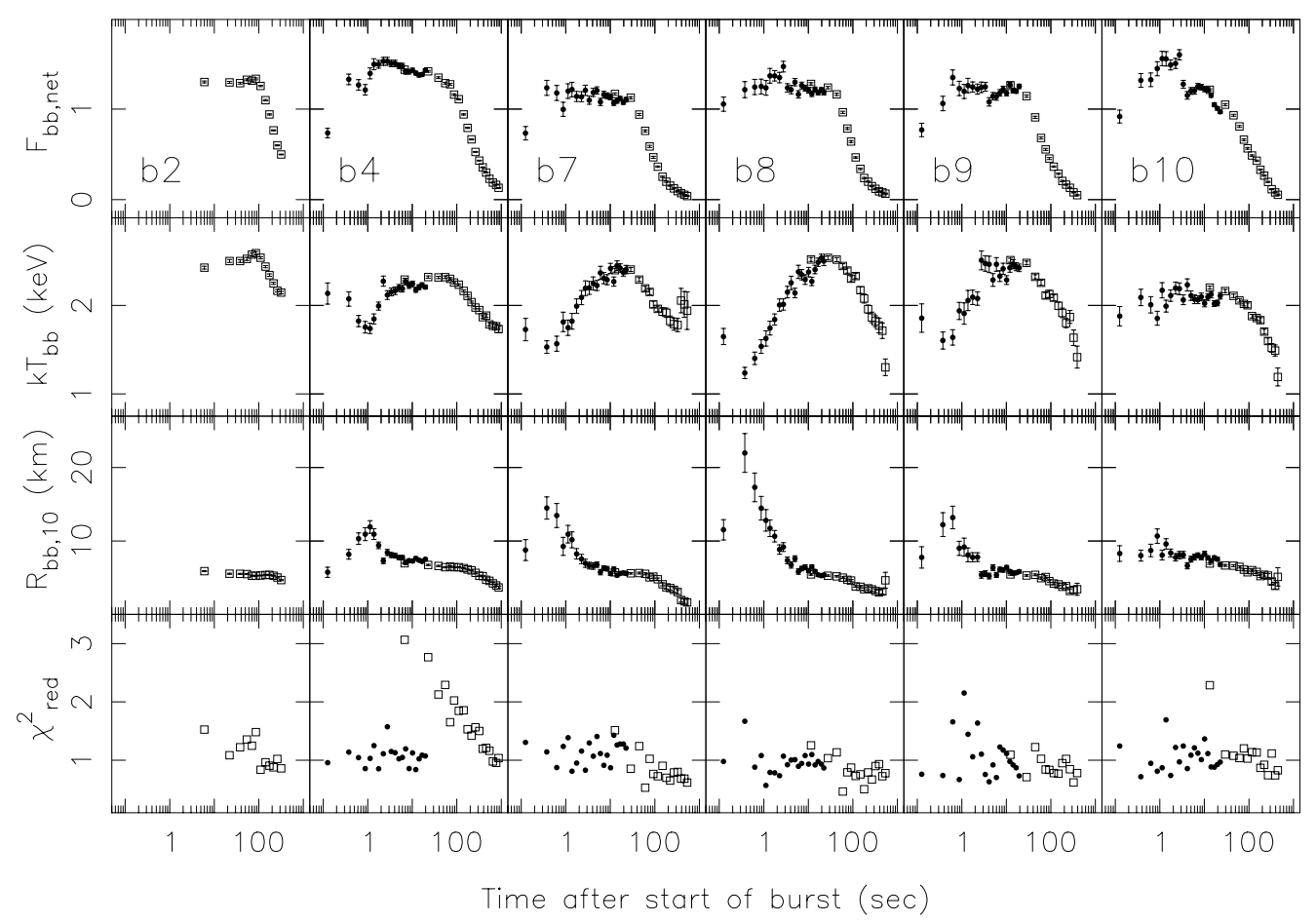

Fig. 12. Spectral fit results for the net burst emission of bursts b2, b4 and b7-b10 plotted on a logarithmic time scale; from top to bottom: bolometric black-body flux, $F_{\mathrm{bb}, \mathrm{net}}$, in $10^{-8} \mathrm{erg} \mathrm{s}^{-1} \mathrm{~cm}^{-2}$, black-body temperature, $k T_{\mathrm{bb}}$, apparent black-body radius, $R_{\mathrm{bb}, 10}$, at $10 \mathrm{kpc}$, and goodness of fit expressed in reduced $\chi^{2}$. The filled circles and open squares represent the fit results of the $0.25 \mathrm{~s}$ and $16 \mathrm{~s}$ spectra, respectively. The data have been logarithmically rebinned for clarity. For the $0.25 \mathrm{~s}$ spectral fits the number of d.o.f. is 18 for burst b4 and 16 for bursts b7-b10. For the $16 \mathrm{~s}$ spectral fits the number of d.o.f. is 44 for bursts b2 and b4, and 37 for bursts b7-b10.

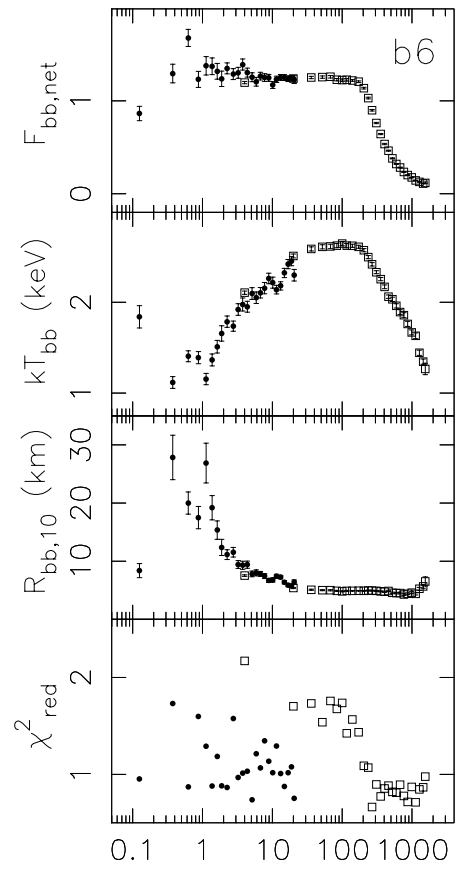

Time after start of burst (sec)

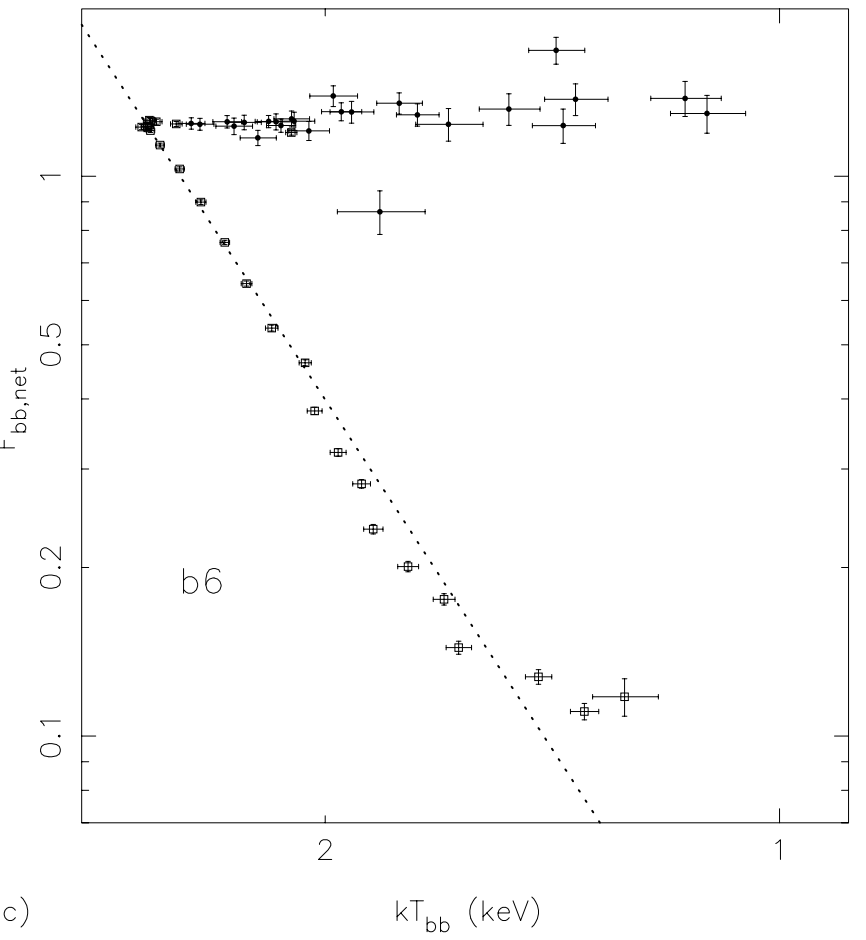

Fig. 13. Left panel: same as Fig. 12, but for burst b6, the longest burst in our sample. Right panel: Bolometric black-body flux $\left(F_{\mathrm{bb}, \text { net }}\right.$ in $\left.10^{-8} \mathrm{erg} \mathrm{s}^{-1} \mathrm{~cm}^{-2}\right)$ versus black-body temperature $\left(k T_{\mathrm{bb}}\right)$ for burst b6. The dotted line represents the fit to the cooling track of the burst for data points with $F_{\mathrm{bb}, \text { net }}>4 \times 10^{-9} \mathrm{erg} \mathrm{s}^{-1} \mathrm{~cm}^{-2}$, see text. Note that $k T_{\mathrm{bb}}$ increases from right to left. For the $0.25 \mathrm{~s}$ and $16 \mathrm{~s}$ spectral fits the number of d.o.f. is 16 and 37 , respectively. 
to $T_{\mathrm{bb}}^{4}$, as expected if the neutron star photosphere radiates as a black-body with constant effective area. Below $F_{\text {bb,net }} \simeq 0.4 \times 10^{-8} \mathrm{erg} \mathrm{s}^{-1} \mathrm{~cm}^{-2}$ the data start to deviate from this relation. The flux-temperature diagrams for the other long bursts are more or less consistent with the behaviour of burst b6, although the exact locations of the vertices between the radius expansion/contraction phase and the cooling phase differ slightly $\left(k T_{\mathrm{bb}} \simeq 2.3\right.$ $\left.2.6 \mathrm{keV}, F_{\mathrm{bb}, \text { net }} \simeq 1.2-1.35 \times 10^{-8} \mathrm{erg} \mathrm{s}^{-1} \mathrm{~cm}^{-2}\right)$. The early parts of the cooling phases for the other long bursts are in most cases also consistent with black-body cooling, although the flux level at which they start to deviate from $F_{\mathrm{bb} \text {,net }} \propto T_{\mathrm{bb}}^{4}$ varies from burst to burst (e.g. $\simeq 1 \times 10^{-8} \mathrm{erg} \mathrm{s}^{-1} \mathrm{~cm}^{-2}$ for burst b4). The slope becomes somewhat steeper below these fluxes for all bursts.

\subsubsection{The radius expansion/contraction track}

As we have shown above, the observed net burst flux is consistent with being constant during the radius expansion/contraction phases. For instance, for burst b6 a constant fit yields $F_{\mathrm{bb}, \text { net }} \simeq 1.24 \times 10^{-8} \mathrm{erg} \mathrm{s}^{-1} \mathrm{~cm}^{-2}$ with a reduced $\chi^{2}$ of 1.4 for 29 d.o.f., excluding the highest flux point. However, for a constant composition and constant (an)isotropy of the radiation, one would expect to see a slight increase in the observed bolometric burst flux with increasing photosphere radius, since the gravitational redshift decreases (see e.g. Lewin et al. 1993). In Fig. 15, we zoom in on the observed radius expansion/contraction phase of burst b6 (note that for $F_{\mathrm{bb} \text {,net }}$ and $T_{\mathrm{bb}}$ we now use a linear scale). Overdrawn is an example of the expected relation between bolometric burst flux and the photospheric radius (continuous line), for "standard" values of the mass of the neutron, $M_{\mathrm{ns}}=1.4 M_{\odot}$, the hydrogen fraction (by mass), $X=0.73$, the spectral hardening factor, $T_{\mathrm{bb}} / T_{\mathrm{eff}, \infty}=1.7$, and high-temperature electron scattering opacity, see Appendix B for details. The curve was normalized so as to give the observed values of $F_{\mathrm{bb} \text {,net }}$ and $T_{\mathrm{bb}}$ at touch down (the leftmost point of the radius expansion/contraction track). In practice this is analogous to solving for the distance and the photospheric radius $R$ at touch down (see Appendix B). We can see that the curve does not follow most of the data points. Taking the lowtemperature electron scattering opacity (but keeping the other parameters at their "standard" values, see above) makes the disagreement even larger (dash-dot-dot-dotted line in Fig. 15). There are three ways to flatten the radius expansion/contraction track, or equivalently, to reduce the gravitational redshift corrections. The most obvious one is to lower the mass of the neutron star; the expected track (dash-dotted line) for $M_{\mathrm{ns}}=0.5 M_{\odot}$ is given. However, such a low-mass neutron star is in not in line with the observed and expected masses for neutron stars (see e.g. Thorsett \& Chakrabarty 1999). A second option is to lower the hydrogen content of the burning material (shown by the dashed line for $X=0$ ). However, the long contraction phase is typical for unstable mixed
H/He-burning (see Sect. 5.4) and not expected for unstable pure He-burning. Moreover, the distance derived at touch down is then rather high $(d=15.2 \mathrm{kpc}$ for $X=0$, see Appendix B). Finally, increasing the hardening factor to $T_{\mathrm{bb}} / T_{\mathrm{eff}, \infty}=2$ also flattens the expected track (dotted line in Fig. 15). This may be the most realistic option, since spectral hardening values of $\sim 2$ are inferred for burst luminosities near the Eddington limit (Babul \& Paczyński 1987; but see Titarchuk 1994, where $T_{\mathrm{bb}} / T_{\mathrm{eff}, \infty}<1$ at very large photospheric radii). We note that the effects of an expanded boundary layer, as discussed in Sect. 5.2.1, may also be of importance here. However, at present it is unclear how to take this into account.

\subsubsection{Burst parameters}

From X-ray spectral fits we can determine, for each burst, the maximum net bolometric black-body flux, $F_{\mathrm{bb}, \max }$, and the total burst fluence (i.e. the integrated net burst flux), $E_{\mathrm{b}}$. These can be used to derive the burst parameters $\gamma=F_{\text {pers }} / F_{\mathrm{bb} \text {, max }}$, where $F_{\text {pers }}$ is the persistent flux flux between 2 and $20 \mathrm{keV}$ (Table 3), and the average burst duration, $\tau=E_{\mathrm{b}} / F_{\mathrm{bb} \text {,max }}$. Note that $F_{\mathrm{bb} \text {,max }}$ may be slightly underestimated due to the finite width of the time bins. The magnitude of this effect depends on how fast the flux varies with time. $E_{\mathrm{b}}$ has been determined by adding up the observed net burst fluxes, $F_{\mathrm{bb}, \text { net }}$, per time bin from the beginning to the estimated end of the burst. Since $F_{\mathrm{bb} \text {,net }}$ decays exponentially it only vanishes finishes at infinite times. To compensate for this we fit the decay with an exponential and determine the "rest" fluence by integrating from the estimated end of the burst up to infinity. The "rest" fluence is large only for burst b2, which was interrupted during its decay (see Fig. 4). We also determined the burst parameter $\alpha$, which is the ratio of the average persistent flux to the time-averaged flux emitted in the bursts, $\alpha=F_{\text {pers }} /\left(E_{\mathrm{b}} / \Delta t\right)$, where $\Delta t$ is the time since the previous burst. For $\alpha$ we can only give lower limits, since the source is not observed during South Atlantic Anomaly passages and earth occultations. For burst b5 we have, however, also assumed that between bursts b4 and b5 no other bursts occurred $(\Delta t=5.77 \mathrm{hr})$. Note that when we derive $\alpha$ we assume that between bursts the persistent luminosity is constant. Since GX $17+2$ is a highly variable source (see e.g. Fig. 1), this is not strictly valid. The burst parameters can be found in Table 4 .

\subsection{Burst position in the $Z$}

The colour-colour diagram (CD) of the data from the three different RXTE gain epochs is shown in Fig. 16, together with the source positions just before the burst (see also Table 2). To determine the position of the source in the CD at the time of the burst, we calculated the soft and hard colour values from $64 \mathrm{~s}$ intervals just before a burst. The three short bursts all occurred when the source was in the lower part of the NB. Among the long bursts, two 
Table 4. Burst parameters.

\begin{tabular}{lccccl}
\hline burst & $F_{\mathrm{bb}, \max }{ }^{a}$ & $E_{\mathrm{b}}{ }^{b}$ & $\tau(\mathrm{s})$ & $\gamma$ & $\alpha$ \\
\hline $\mathrm{b} 1$ & $1.59 \pm 0.06$ & $5.38 \pm 0.07$ & $3.39 \pm 0.14$ & $1.3 \pm 0.2$ & $\gtrsim 700$ \\
$\mathrm{~b} 2^{c}$ & $1.33 \pm 0.01$ & $356.4 \pm 0.7$ & $267 \pm 2$ & $1.7 \pm 0.4$ & $\gtrsim 15$ \\
$\mathrm{~b} 3$ & $0.79 \pm 0.05$ & $5.54 \pm 0.09$ & $7.0 \pm 0.5$ & $2.4 \pm 0.4$ & $\gtrsim 250$ \\
$\mathrm{~b} 4$ & $1.59 \pm 0.06$ & $430.2 \pm 1.1$ & $271 \pm 11$ & $1.2 \pm 0.2$ & $\gtrsim 10$ \\
$\mathrm{~b} 5$ & $1.46 \pm 0.07$ & $5.26 \pm 0.07$ & $3.60 \pm 0.17$ & $1.6 \pm 0.2$ & $\gtrsim 100$ \\
$\mathrm{~b} 5$ & & & & $9000 \pm 1300^{d}$ \\
$\mathrm{~b} 6$ & $1.68 \pm 0.09$ & $664.1 \pm 1.9$ & $396 \pm 22$ & $1.5 \pm 0.1$ & $\gtrsim 1$ \\
$\mathrm{~b} 7$ & $1.36 \pm 0.08$ & $143.3 \pm 1.0$ & $106 \pm 6$ & $1.8 \pm 0.1$ & $\gtrsim 5$ \\
$\mathrm{~b} 8$ & $1.53 \pm 0.08$ & $176.4 \pm 1.1$ & $115 \pm 6$ & $1.6 \pm 0.3$ & $\gtrsim 25$ \\
$\mathrm{~b} 9$ & $1.50 \pm 0.08$ & $137.6 \pm 1.0$ & $92 \pm 5$ & $1.6 \pm 0.3$ & $\gtrsim 25$ \\
$\mathrm{~b} 10$ & $1.65 \pm 0.08$ & $169.0 \pm 0.9$ & $102 \pm 5$ & $1.2 \pm 0.2$ & $\gtrsim 15$ \\
\hline${ }^{a}$ Bolometric peak net-burst black-body flux in $10^{-8} \mathrm{erg} \mathrm{s}^{-1} \mathrm{~cm}^{-2}$. \\
${ }^{b}$ Burst fluence in 10 $0^{-8}$ erg cm ${ }^{-2}$. \\
${ }^{c}$ Using the $16 \mathrm{~s}$ spectral fit results. \\
${ }^{d}$ Assuming that between burst b4 and b5 no other bursts occurred.
\end{tabular}

occurred in the lower part of the $\mathrm{HB}$, four in the NB, and one close to the NB/FB vertex. During the observations in $1996-2000$ GX $17+2$ spent about $28 \%$ of its time in the $\mathrm{HB}, 44 \%$ of its time in the $\mathrm{NB}$, and $28 \%$ in the FB. It thus seems that the NB is overpopulated, whereas the FB is underpopulated with bursts (i.e. none occurred). However, by assuming that bursts have an equal chance to occur at each instant independent of branch, this result is statistically not significant (4\% probability seeing 7 out of 10 bursts in the NB, and $7 \%$ of seeing none out of 10 bursts in the FB). Our conclusion does not change much if we include the EXOSAT/ME results reported by Kuulkers et al. (1997); for $260 \mathrm{ksec}$ of observing time, GX $17+2$ spent $11 \%$ of the time in the HB, $66 \%$ in the NB and $23 \%$ in the $\mathrm{FB}$, with 3 bursts occurring in the NB and 1 in the (lower part of the) FB. This leads to total probabilities of $7 \%$ of seeing 10 out of 14 bursts in the NB, and $6 \%$ of seeing only 1 out of 14 bursts in the FB.

\section{Search for burst oscillations}

Following the method described by Leahy et al. (1983; see also Vaughan et al. 1994) we defined a $99 \%$ confidence level above which powers are regarded to be due to a real signal. This level depends on the number of trials, which basically is the number of independent frequency bins that are examined. In our search this number is $\simeq 2 \times 10^{7}$ which leads to a trigger level of 42.8 in the Leahy et al. (1983) normalization. This level was never reached, indicating that no coherent oscillations were detected in any of the bursts. We inspected the frequencies of the highest observed powers. These powers occurred at apparently random frequencies, consistent with the idea that they are due to random fluctuations.

Since no powers were found above the trigger level, only upper limits to the strength of possible burst oscilla-

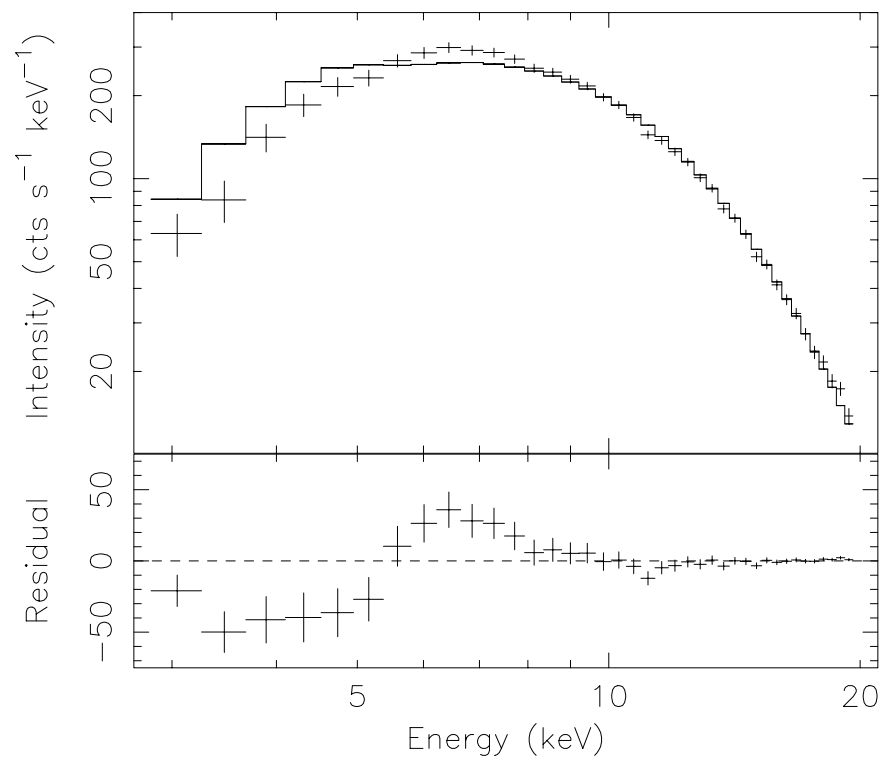

Fig. 14. Top panel: average observed net burst (i.e. total source minus persistent emission) spectrum during the flat top of burst b6, i.e. from 27 to $187 \mathrm{~s}$ after the start of the burst. A black-body fit it shown $\left(k T_{\mathrm{bb}}=2.658 \pm 0.015 \mathrm{keV}\right.$, $R_{\mathrm{bb}, 10}=4.65 \pm 0.06 \mathrm{~km}, \chi_{\mathrm{red}}^{2}=2.4$ for 37 d.o.f.). Bottom panel: residuals after subtracting the best fit black-body model from the observed spectrum.

tions can be given (see van der Klis 1989; Vaughan et al. 1994). We used the (maximum) observed powers to determine a $99 \%$ confidence upper limit to a true signal power. These upper limit powers were then converted into the full amplitude of an assumed sinusoidal signal ${ }^{3}$, which is given

${ }^{3}$ If a sinusoidal signal is given by $y=a \sin x$, the full amplitude is defined as $A=2 a$. 


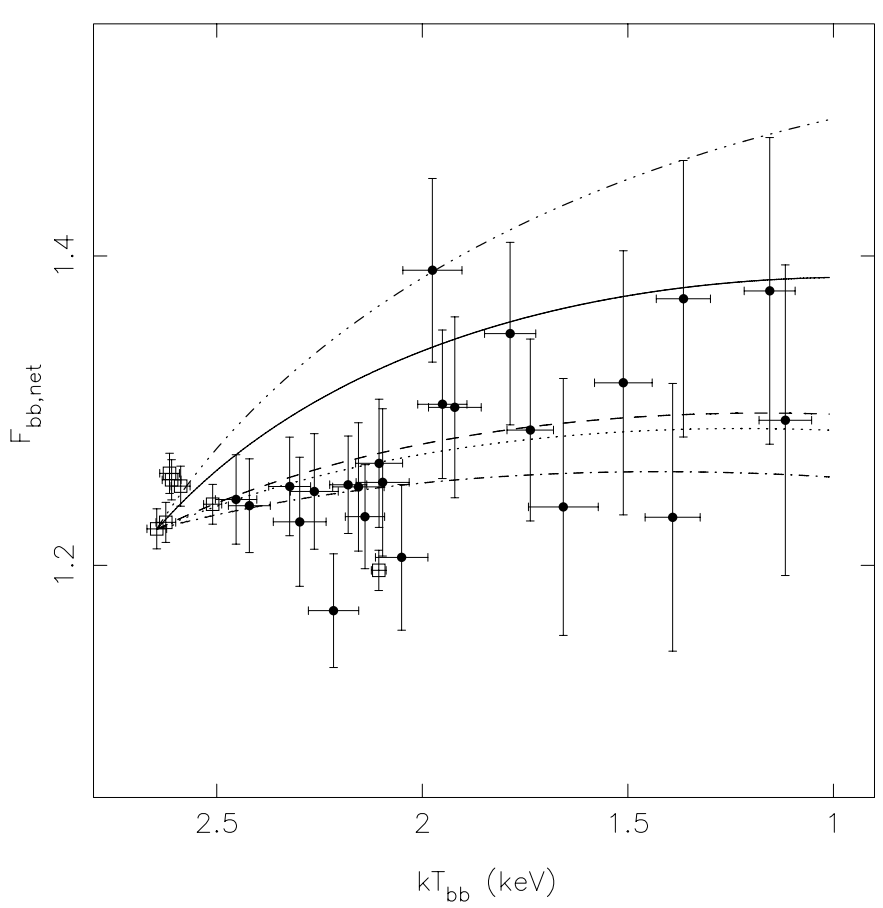

Fig. 15. Same as the right panel Fig. 13, but only the radius expansion/contraction phase is shown (note that the highest flux point lies outside the plot boundaries). The five lines correspond to expected tracks in the same diagram for different sets of parameters, see text.

by:

$A=1.61 \sqrt{\frac{P_{\mathrm{ul}}}{N}}\left[\operatorname{sinc}\left(\frac{\pi}{2} \frac{\nu}{\nu_{\mathrm{Nyq}}}\right)\right]^{-1}$,

where $P_{\mathrm{ul}}$ is the (upper limit) signal power (Vaughan et al. 1994; Groth 1975), $N$ the number of photons used in the power spectrum, $\nu$ the frequency at which the power was found, and $\nu_{\mathrm{Nyq}}$ the Nyquist frequency. Table 5 gives the upper limits on $A$ for all bursts - each burst was divided into four parts: $5 \mathrm{~s}$ before the rise, the rise, the peak, and decay ( $=$ end of peak $+2 \times t_{\exp }$ ). Note that $A$ is a factor $\sqrt{2}$ larger than the rms amplitude that is often quoted.

The values of the upper limits are not very constraining. Only those obtained from the $2 \mathrm{~s}$ power spectra in the $2-60 \mathrm{keV}$ band are well below the strongest values observed in some of the atoll sources (see e.g. Strohmayer 1998, 2001).

\section{Discussion}

\subsection{X-ray burst properties}

Of the ten X-ray bursts from GX $17+2$ seen so far with the RXTE/PCA, three are rather short ( $\simeq 10$ s), whereas the other seven have durations of $\simeq 6-25 \mathrm{~min}$. They all show evidence for cooling of the neutron star photosphere during the decay, confirming previous results. The burst durations found are in the range seen previously (Kahn \& Grindlay 1984; Tawara et al. 1984c; Sztajno et al. 1986;
Kuulkers et al. 1997). Using the time-resolved X-ray spectral fits (see Sect. 5.2) we determined the burst fluence, $\tau$ (a characterisation of the burst decay time), $\gamma$ (the net brightness of the burst with respect to the persistent level) and $\alpha$ (the ratio between the total net burst flux and total persistent flux in between bursts). Since not all these parameters were given for GX $17+2$ bursts observed previously, we (re-)determined these values from the information given in the various papers. This is explained in Appendix A and the results are given in Table A.1. Again, our values for the burst parameters for both the short and long bursts are comparable to those for the bursts observed previously. Since observations with RXTE are interrupted by either SAA passages or Earth occultations, and the fact that the length of each 'continuous' observation was limited ( $\sim$ hours) we could not determine the time interval between bursts. Our lower limits on $\alpha$ are, therefore, rather low. However, from the bursts observed previously, it is found that $\alpha$ always exceeds $\sim 1000$. This means that most of the accreted material is being burned continuously. One of our short bursts occurred about $5.8 \mathrm{hr}$ after a long burst. So far this is the shortest recurrence time encountered for a burst in GX $17+2$. The fluences of the bursts from GX $17+2$ are either $\simeq 5.5 \times 10^{-8} \mathrm{erg} \mathrm{cm}^{-2}$ (short bursts) or $140-670 \times 10^{-8} \mathrm{erg} \mathrm{cm}^{-2}$ (long bursts). At a distance of $10 \mathrm{kpc}$ (but see Sect. 5.3) these correspond to total energies of $6.5 \times 10^{38} \mathrm{erg}$ and $2-8 \times 10^{40} \mathrm{erg}$, respectively, in the range seen for type I bursts (see e.g. Lewin et al. 1993).

One of the short bursts was rather faint, with a peak intensity which was a factor of $\simeq 1.25$ lower than for the other bursts, and displayed two peaks in the light and bolometric flux curves. This burst resembles the (relatively) weak double-peaked bursts seen from 4U 1636-53 (Sztajno et al. 1985; Fujimoto et al. 1988). Such bursts are clearly not radius expansion/contraction events, but are instead thought to be due to the mixing of fresh material into the unstably burning layer; such mixing may be caused by shear instabilities in the outer envelope of the neutron star (Fujimoto et al. 1988).

We show for the first time that almost all of the long bursts of GX $17+2$ have episodes of radius expansion and contraction of the neutron star photosphere. In all cases the expansion and initial contraction occur during the first few seconds of the bursts, but the final contraction phase duration varies between $\simeq 20$ s and $\simeq 180$ s. During the expansion/contraction phase the net burst flux remains constant, presumably at the Eddington value. Long contraction phases might be caused by long phases of unstable hydrogen burning, whose time scale is limited by $\beta$-decays, up to $\sim 100$ s after the burst has started (see e.g. Bildsten 1998, 2000, and references therein). The maximum apparent radius at $10 \mathrm{kpc}, R_{\mathrm{bb}, 10}$, reached during the expansion episodes is $\simeq 30 \mathrm{~km}$. Corrected for gravitational redshift effects and spectral hardening (see Appendix B), the maximum photospheric radius $R$ (as measured by a local observer) is $\simeq 90-125 \mathrm{~km}$. We do not see evidence for a change in the cut-off power-law component above 
Table 5. Upper limits on burst oscillations.

\begin{tabular}{|c|c|c|c|c|c|c|c|c|c|c|c|}
\hline \multirow[t]{3}{*}{ burst } & \multirow[t]{3}{*}{$\phi^{a}$} & \multicolumn{4}{|c|}{ upper limits on fractional amplitude ${ }^{b}$} & \multirow[t]{3}{*}{ burst } & \multirow[t]{3}{*}{$\phi^{a}$} & \multicolumn{4}{|c|}{ upper limits on fractional amplitude ${ }^{b}$} \\
\hline & & $0.25 \mathrm{~s}$ & $2 \mathrm{~s}$ & $0.25 \mathrm{~s}$ & $2 \mathrm{~s}$ & & & $0.25 \mathrm{~s}$ & $2 \mathrm{~s}$ & $0.25 \mathrm{~s}$ & $2 \mathrm{~s}$ \\
\hline & & $2-60 \mathrm{keV}$ & $2-60 \mathrm{keV}$ & $8-20 \mathrm{keV}$ & $8-20 \mathrm{keV}$ & & & $2-60 \mathrm{keV}$ & $2-60 \mathrm{keV}$ & $8-20 \mathrm{keV}$ & $8-20 \mathrm{keV}$ \\
\hline \multirow[t]{4}{*}{$\mathrm{b} 1^{c}$} & $\mathrm{~b}$ & 0.28 & 0.12 & $>1.0$ & 0.67 & \multirow[t]{4}{*}{ b6 } & $\mathrm{b}$ & 0.41 & 0.14 & 0.70 & 0.28 \\
\hline & $\mathrm{r}$ & 0.23 & 0.07 & $>1.0$ & 0.34 & & $\mathrm{r}$ & 0.28 & 0.09 & 0.49 & 0.24 \\
\hline & $\mathrm{p}$ & 0.25 & 0.09 & $>1.0$ & 0.45 & & $\mathrm{p}$ & 0.40 & 0.15 & 0.63 & 0.24 \\
\hline & $\mathrm{d}$ & 0.27 & 0.11 & $>1.0$ & 0.60 & & d & 0.45 & 0.16 & 0.89 & 0.30 \\
\hline \multirow[t]{4}{*}{ b2 } & b & 0.34 & 0.10 & 0.66 & 0.26 & \multirow[t]{4}{*}{ b7 } & b & 0.47 & 0.16 & 0.78 & 0.26 \\
\hline & $\mathrm{r}$ & 0.19 & 0.07 & 0.51 & 0.20 & & $\mathrm{r}$ & 0.30 & 0.08 & 0.58 & 0.15 \\
\hline & $\mathrm{p}$ & 0.29 & 0.10 & 0.55 & 0.20 & & $\mathrm{p}$ & 0.36 & 0.13 & 0.63 & 0.23 \\
\hline & $\mathrm{d}$ & 0.33 & 0.11 & 0.68 & 0.26 & & d & 0.46 & 0.16 & 0.80 & 0.34 \\
\hline \multirow[t]{4}{*}{ b3 } & b & 0.36 & 0.13 & 0.77 & 0.25 & \multirow[t]{4}{*}{ b8 } & b & 0.41 & 0.14 & 0.74 & 0.26 \\
\hline & $\mathrm{r}$ & 0.21 & 0.08 & 0.51 & 0.17 & & $\mathrm{r}$ & 0.33 & 0.10 & 0.56 & 0.20 \\
\hline & $\mathrm{p}$ & 0.32 & 0.12 & 0.64 & 0.24 & & $\mathrm{p}$ & 0.37 & 0.14 & 0.59 & 0.24 \\
\hline & $\mathrm{d}$ & 0.32 & 0.12 & 0.71 & 0.25 & & d & 0.43 & 0.16 & 0.72 & 0.27 \\
\hline \multirow[t]{4}{*}{$\mathrm{b} 4$} & b & 0.37 & 0.14 & 0.76 & 0.27 & \multirow[t]{4}{*}{ b9 } & b & 0.41 & 0.13 & 0.77 & 0.30 \\
\hline & $\mathrm{r}$ & 0.30 & 0.09 & 0.62 & 0.23 & & $\mathrm{r}$ & 0.25 & 0.09 & 0.56 & 0.22 \\
\hline & $\mathrm{p}$ & 0.29 & 0.11 & 0.55 & 0.20 & & $\mathrm{p}$ & 0.38 & 0.14 & 0.63 & 0.22 \\
\hline & $\mathrm{d}$ & 0.38 & 0.13 & 0.75 & 0.27 & & d & 0.45 & 0.17 & 0.78 & 0.28 \\
\hline \multirow[t]{4}{*}{ b5 } & $\mathrm{b}$ & 0.33 & 0.11 & 0.63 & 0.22 & \multirow[t]{4}{*}{ b10 } & b & 0.45 & 0.19 & 0.67 & 0.34 \\
\hline & $\mathrm{r}$ & 0.24 & 0.09 & 0.42 & 0.17 & & $\mathrm{r}$ & 0.34 & 0.09 & 0.48 & 0.23 \\
\hline & $\mathrm{p}$ & 0.27 & 0.10 & 0.39 & 0.17 & & $\mathrm{p}$ & 0.38 & 0.14 & 0.51 & 0.26 \\
\hline & d & 0.28 & 0.10 & 0.57 & 0.22 & & $\mathrm{~d}$ & 0.48 & 0.18 & 0.68 & 0.34 \\
\hline
\end{tabular}

${ }^{a}$ Phase of burst profile: $\mathrm{b}=$ before burst, $\mathrm{r}=$ rise, $\mathrm{p}=$ peak, $\mathrm{d}=$ decay.

${ }^{b} 99 \%$ confidence upper limits in the $50-2000 \mathrm{~Hz}$ frequency range.

${ }^{c} 99 \%$ confidence upper limits in the $2-60 \mathrm{keV}$ energy band are between $50-250 \mathrm{~Hz}$. The high-energy band used is $13.5-20 \mathrm{keV}$.

$\sim 10 \mathrm{keV}$ when the photosphere expands up to these radii. This suggests that either the environment in between the neutron star surface and expanded photosphere at $\simeq 90$ $125 \mathrm{~km}$ is involved in producing the high-energy radiation but was not affected, or the inner disk and corona which presumably produce the high-energy radiation do not extend down to the neutron star. A possibility is that the magnetospheric radius extends out to at least these radii, disrupting the disk and corona near that point. Note that the radius range of $\simeq 90-125 \mathrm{~km}$ overlaps with the range of magnetospheric radii expected for Z sources $(\sim 10-100 \mathrm{~km}$, see Ghosh \& Lamb 1992). However, at the high mass accretion rates inferred for $\mathrm{GX} 17+2$ the disk is expected to extend down to close to the neutron star (see Popham \& Sunyaev 2001). For a more detailed discussion of the different emission regions we refer to the next subsection.

Long (>minutes) X-ray bursts have been seen in many other sources (see, in order of decreasing duration, Hoffman et al. 1978b, Lewin et al. 1984, $\simeq 25 \mathrm{~min}$ : 4U 1708-23; Swank et al. 1977, ¿10 min: 4U 1724-307; Tawara et al. 1984a,b, ¿4.5 min: 3A 1715-321; Kaptein et al. 2000, ¿3.3 min: 1RXS J171824.2-402934; van Paradijs et al. 1990, ¿2.7 min: $4 \mathrm{U} 2129+11)$. The shapes of the light curves of these bursts at different energies are very similar to the long bursts of GX $17+2$, i.e. fast (several seconds) rise and peaked emission at low energies and relatively slower rise at high energies. They also have rather long episodes of contraction, i.e. long episodes of Eddington fluxes, up to $\simeq 300 \mathrm{~s}$ (see e.g. Lewin et al. 1984). Moreover, the curves for $T_{\mathrm{bb}}$ and $R_{\mathrm{bb}}$ as a function of time show similar shapes.

The difference, however, between the long bursts of GX $17+2$ and those in the other sources is that "precursors" are present in the light curves before almost all of the long bursts from other sources. Note that these are not real precursors, but indicate very large radius expansion $\left(R_{\mathrm{bb}}>100 \mathrm{~km}\right)$. No emission is then seen because of the very low effective temperatures reached; the peak of the emission is shifted to UV wavelengths. Thus the $\mathrm{X}$-ray burst light curve is interrupted by a gap of no $\mathrm{X}$ ray emission leading to an apparent precursor (see Lewin et al. 1984). The rise to maximum radius in those sources is on the order of several seconds. In GX $17+2$ the maximum value of $R_{\mathrm{bb}}$ is a few times the neutron star radius and the expansion lasts typically only a second. The most important difference, however, is that the persistent emission just before the long bursts in other sources is $\sim 1 \%$ of the Eddington value, i.e. a factor of $\sim 100$ lower than in GX 17+2 (see also Sect. 5.4). 

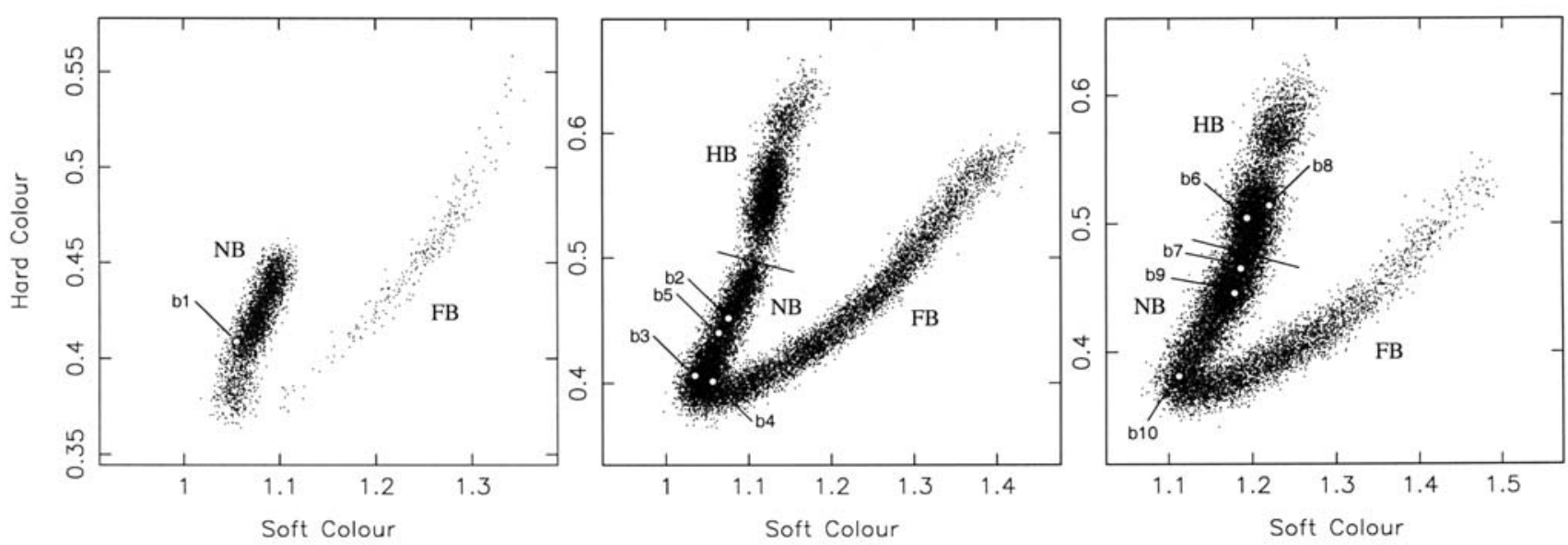

Fig. 16. Colour-colour diagrams of the RXTE/PCA observations of GX $17+2$ (after Homan et al. 2001). The left, middle and right panels refer to the observations done during RXTE gain epochs 1, 3 and 4, respectively. The soft colour is defined as the count rate ratio in the $4.8-7.2 \mathrm{keV}$ to the $3.0-4.8 \mathrm{keV}$ energy bands for the epoch 1 data, in the $4.8-7.3 \mathrm{keV}$ to the $3.0-4.8 \mathrm{keV}$ energy bands for the epoch 3 data, and in the 4.6-7.1 keV to the 3.0-4.6 keV energy bands for the epoch 4 data. The hard colour is defined as the count rate ratio in the $10.6-19.7 \mathrm{keV}$ to the $7.2-10.6 \mathrm{keV}$ energy bands for the epoch 1 data, in the $10.5-19.6 \mathrm{keV}$ to the $7.3-10.5 \mathrm{keV}$ energy bands for the epoch 3 data, and in the $10.5-19.5 \mathrm{keV}$ to the $7.1-10.5 \mathrm{keV}$ energy bands for the epoch 4 data. Each point represents an average of $16 \mathrm{~s}$. We have indicated the position of the source during $64 \mathrm{~s}$ intervals just before a burst (indicated by b1, b2, .., b10, in chronological order), and the three limbs of the Z (HB, NB, FB). Since the $\mathrm{HB}$ is not clearly distinguishable from the NB in this figure, we have indicated the approximate HB/NB vertex by a line.

We note that even longer X-ray bursts do exist, which last for several hours (Cornelisse et al. 2000, 2002; Wijnands 2001; Strohmayer \& Brown 2001; Kuulkers 2001; Kuulkers et al. 2002). Their recurrence time is much longer than normal type I bursts ( $\gtrsim 7.5$ days, Cornelisse et al. 2002; $\lesssim 4.8$ yrs, Wijnands 2001), and they seem to occur at persistent luminosites which are a factor of $\sim 0.1$ 0.2 of the Eddington limit.

\subsection{Black-body spectral fits}

\subsubsection{Decoupled persistent and burst emission}

It has previously been pointed out (van Paradijs \& Lewin 1986; see also Sztajno et al. 1986) that, if the persistent emission before the burst contains a spectral component which originates from the same regions as the burst emission, this should be taken into account in the spectral analysis of X-ray bursts. This is especially the case when the neutron star is accreting matter at high rates such as thought to be the case in $\mathrm{Z}$ sources, making the neutron star hot and radiate soft (black-body like) emission. "Standard" X-ray burst spectral analyses (modeling the net-burst emission by black-body radiation) then results in systematic errors in the spectral parameters, especially during the end of the burst when the net-burst luminosity is small. It was therefore suggested to model the total burst emission using a two-component approach, i.e. with a soft component (usually a black body) and a hard component (fixed at the parameters found for the persistent emission). The former component is thought to contain all emission from the neutron star photosphere, while the latter component is assumed to be from the accretion disk and not affected by the prompt burst. However, we find that using this two-component spectral fitting approach does not give satisfactory results. Instead, the "standard" burst analysis, which assumes the persistent emission continues unchanged, yield better fits.

This suggests that the persistent emission is unaffected during the burst and that the persistent black-body component (which is not always present) does not arise from the same region as the burst emission, contrary to what was previously assumed. We find that during the initial exponential decay phase of the bursts the neutron star photosphere shows pure black-body cooling. During that stage the apparent black-body radius stays constant at 5 $6 \mathrm{~km}$ (at $10 \mathrm{kpc}$ ). Comparing these radii with the apparent radii of the black-body component of the persistent emission (if present) indicates that the persistent black-body component originates from a larger area than that of the burst emission (see Fig. 8). The inner part of the accretion disk or an expanded boundary layer are the most obvious sites for this persistent soft component. A recent study of X-ray spectra from LMXBs with luminosities spanning several orders of magnitude has shown that the persistent black-body emission likely does not arise in (part of) the accretion disk (Church \& Bałucińska-Church 2001). Instead at very high accretion rates (near Eddington values) the accretion disk boundary layer around the neutron star expands radially, with radial extents larger than one stellar radius (Popham \& Sunyaev 2001). Moreover, at these high rates the expected spectrum of the boundary layer strongly resembles a black-body. The fact that we see black-body emission in the persistent spectra with apparent radii which are larger (factor $\sim 2-3$ ) than the neutron star (see also Church \& Bałucińska-Church 2001) is in 
line with this. Still, one would expect that such a boundary layer would be affected by the bursts (see Popham \& Sunyaev 2001), of which we do not see clear evidence from our spectral fits. We note, however, that Homan et al. (2001), who studied the properties of the NB and FB quasi-periodic oscillations (NB/FB QPO or NBO/FBO) during bursts b4 and b10, found that during the bursts the absolute amplitude of these QPOs decreased significantly. This suggests that the inner accretion flow is affected by the increase in the radiation from the neutron star, contrary to what our spectral results indicate. Apparently the QPO mechanism of the NBO/FBO is much more sensitive to the radiation field than the bulk flow of matter itself. This is in accordance with models for NBO/FBO (Fortner et al. 1989), which require a delicate balance between the radiation field and the accretion flow, that might easily be disrupted during X-ray bursts.

\subsubsection{Deviations from black-body emission}

During the radius expansion/contraction phase blackbody emission does not provide a good description of our observed net-burst spectra, leaving a slight excess in emission between $\simeq 5.5-10 \mathrm{keV}$ and lack of emission between $\simeq 3-5.5 \mathrm{keV}$ (Fig. 11). Such systematic deviations have been observed before, especially during radius expansion/contraction phases. For example, spectra obtained with the Large Area Counter onboard Ginga during the long burst of $4 \mathrm{U} 2129+11$ (van Paradijs et al. 1990) show remarkably similar deviations at the same energies as we see in GX $17+2$. The persistent mass accretion in $4 \mathrm{U} 2129+11$ is inferred to be a factor of $\sim 100$ lower than for GX $17+2$. This suggests that the radiation properties of the photospheres are the same when they are radiating near the Eddington luminosity, which is independent of the mass accretion rate. As noted by van Paradijs et al. (1990), the "bumpiness" of the residuals point to the presence of relatively narrow-band spectral features, rather than the residuals being due to broad-band spectral hardening at Eddington luminosities (see also Strohmayer \& Brown 2001).

From our "standard" spectral analysis, we find that the apparent black-body radii decrease near the end of the decay of the bursts. This was also inferred from the EXOSAT/ME observations of GX $17+2$ (Sztajno et al. 1986), and has been seen in other sources as well (e.g. Chevalier \& Ilovaisky 1990). If this were due to a real decrease in the burst emitting area one might expect to see burst oscillations due to the spinning neutron star. We do not see evidence for this. If the expanded boundary layer reacts to its changing environment on the same time scale as the burst itself then variations in this layer's structure could also play a role here.

This observed behaviour resembles the systematic effects described by van Paradijs \& Lewin (1986), which occurs near the end of the bursts in the presence of a persistent hot neutron star component. However, we have argued that the persistent black body component does not contribute to the burst emission, so this is not a viable explanation. Sztajno et al. (1986) argued that this effect also can not be due to spectral hardening, since that would lead to an apparent radius increase with decreasing $T_{\mathrm{bb}}$. Their argument was based on the assumption that the spectral hardening decreases with decreasing temperatures (and therefore decreasing burst luminosity; see also Sztajno et al. 1985). However, later model calculations have shown that in fact spectral hardening increases again whenever the burst luminosity drops below a certain value $\left(L_{\mathrm{bb}} \lesssim 0.2 L_{\mathrm{Edd}}\right.$, e.g. London et al. 1986; Ebisuzaki 1987). For an assumed constant size of the emission region during the cooling stage (presumably the whole neutron star surface), the apparent radii will then become smaller as $T_{\mathrm{bb}}$ decreases, consistent with what is observed.

\subsection{The distance to $G X 17+2$ and the persistent emission}

Assuming that during the expansion and contraction phase the net-burst luminosity equals the Eddington luminosity we can estimate the distance to GX17+2. As noted before (Lewin et al. 1993; see also recent discussion by Kuulkers \& van der Klis 2000), this is not without problems. Assuming (see Appendix B) a neutron star with a canonical mass of $1.4 M_{\odot}$, using the electron scattering opacity, $\kappa$, for cosmic composition in the low-temperature limit, we obtain $L_{\mathrm{Edd}}=4 \pi c G M / \kappa=2 \times 10^{38} \mathrm{erg} \mathrm{s}^{-1}$. For the observed values of $F_{\text {bol }} \simeq 1.2-1.3 \times 10^{-8} \mathrm{erg} \mathrm{s}^{-1} \mathrm{~cm}^{-2}$ this leads to distances of $d \simeq 11.4-11.9 \mathrm{kpc}$ (assuming isotropic radiation and no spectral hardening, i.e. $T_{\text {eff }, \infty}=$ $\left.T_{\mathrm{bb}}\right)$. Close to the neutron star, however, one still has to take into account gravitational redshift effects. We therefore estimated the distance by solving the relevant equations, for the explanation of which we refer the reader to Appendix B. Correcting for gravitational redshift effects, taking the same 'standard' parameters as above, using values of $\kappa$ appropriate for very high temperatures, and correcting for spectral hardening $\left(T_{\mathrm{bb}} / T_{\mathrm{eff}, \infty}=1.7\right)$ we found distances between $10.8-12.0 \mathrm{kpc}$, with an average of $\mathrm{d} \simeq 11.3 \mathrm{kpc}$ (again see Appendix B for details). If the burst emission is highly anisotropic $(\xi \simeq 2)$ we derive $d \simeq 8 \mathrm{kpc}$, see below. We note that these estimates have systematic uncertainties of the order of $30 \%$ or so (see Lewin et al. 1993; see also Kuulkers \& van der Klis 2000, and Appendix B). At touch down the radius of the photosphere, $R$, presumably equals the radius of the neutron star surface. We find values of $R \simeq 12-20 \mathrm{~km}$, where the range arises from the different assumptions in deriving these radii (Appendix B).

One can also use the radius expansion events to estimate the persistent luminosity in Eddington units, without the need of knowing the distance to the source, mass of the neutron star, etc. However, for the long bursts we find that the total emission before/after the burst is a up to factor of $\sim 2$ larger than the net-burst emission during 
expansion/contraction phase. If indeed the maximum netburst flux equals the Eddington flux, this implies that the persistent flux is up to 2 times the Eddington flux. From the models characterising the behaviour of $\mathrm{Z}$ sources it is inferred that on the $\mathrm{HB}, \mathrm{NB}$ and $\mathrm{FB}$, the mass accretion rates (and therefore luminosity) are just below, at or just above Eddington values, respectively (e.g. Hasinger 1987; Lamb 1989; Hasinger et al. 1990). The only way out is that the net burst emission is (highly) anisotropic compared to the persistent emission. Since the true luminosity values should be more or less comparable, one thus infers an anisotropy factor of $\sim 2$ for the net burst emission. This cannot be ascribed to localized emission on the neutron star (see also Sect. 5.4), but might be due to part of the burst emission being hidden behind a puffedup inner accretion disk. In the case of highly anisotropic burst emission $(\xi \simeq 2)$ the distance estimate to GX $17+2$ is reduced to $\simeq 8 \mathrm{kpc}$ (see Appendix $\mathrm{B}$ ). This estimate is comparable to that derived for other similar X-ray sources (e.g. Christian \& Swank 1997; Schulz 1999). We note that anisotropic burst emission may also explain the apparent inconsistency in the distance to $\mathrm{Cyg} \mathrm{X}-2$ from a radius expansion burst, i.e. $\simeq 11.6 \mathrm{kpc}$ (Smale 1998; for a $1.9 M_{\odot}$ neutron star, peak photospheric radius of $26 \mathrm{~km}$, cosmic abundances, low temperature opacity, no spectral hardening; see also Appendix B), and that derived from optical measurements, i.e. $\simeq 7.2 \mathrm{kpc}$ (Orosz \& Kuulkers 1999). However, for Cyg X-2 the persistent flux before the burst was of the same order as the net peak burst flux.

\subsection{X-ray bursts and (extreme) mass accretion rates}

We found no significant correlation between the burst parameters and the position of the source in the Z-track at the time of the bursts, i.e. both long and short bursts may occur at similar mass accretion rates. We find that no X-ray bursts occurred when the source was on the FB, despite considerable coverage compared to the other branches of the Z. However, this result is not sufficiently significant to exclude the possibility that bursts occur on the FB as frequently as elsewhere. We do note that the absence of bursts on the FB is consistent with theoretical expectations (see Sect. 1; e.g. Bildsten 1998, 2000), as the mass accretion rate on the FB is thought to be superEddington (e.g. Hasinger 1987; Lamb 1989; Hasinger et al. 1990).

At near-Eddington accretion rates, long bursts are expected from X-ray burst theory (see Sect. 1; see also van Paradijs et al. 1988). These are thought to be due to mixed $\mathrm{H} / \mathrm{He}$ burning, triggered by thermally unstable $\mathrm{He}$ ignition. The fast rise and the short radius expansion part of the long bursts resemble pure helium flashes which last on the order of $5-10 \mathrm{~s}$, although in the case of GX17+2 the recurrence times of these bursts are on the order of a day instead of the expected hours for typical pure helium flashes. This flash triggers the long phase of unstable mixed $\mathrm{H} / \mathrm{He}$ burning. Our analysis provides the first example of the existence of such long bursts at high (near-Eddington) accretion rates. However, GX $17+2$ also displays short $(\simeq 10 \mathrm{~s})$ bursts, which are not expected at high accretion rates. Similarly, Cyg X-2, also accreting at near-Eddington values, displays rather short bursts $(\simeq 5 \mathrm{~s})$. Such short bursts (presumably He flashes) are only expected for accretion rates which are a factor of 20-100 lower.

Since it is not the global mass accretion rate that matters, but the mass accretion rate per unit area (e.g. Marshall 1982; see also Bildsten 2000), it might be that at different times the area which accumulates most of the accreted matter differs, giving rise to short and long bursts. However, if the areas are relatively small, pulsations are expected due to the neutron star rotation (unless the accreted matter is distributed symmetrically along the rotation axis, such as in an equatorial belt). We do not see any evidence for this.

The properties of X-ray bursts depend not only on the mass accretion rate (per unit area), but also on the temperature of the neutron star envelope and composition of the accreted material (e.g. Fushiki \& Lamb 1987; Taam et al. 1996; see also Bildsten 1998, 2000; Lamb 2000). At high accretion rates and high envelope temperatures the combined He flash and mixed $\mathrm{H} / \mathrm{He}$ burning occurs; however at high accretion rates and low envelope temperatures only He flashes may occur (see Lamb 2000). The difference in envelope temperature at different times may indeed explain the occurrence of the two types of bursts, and it would be worthwhile to explore this further. It is not clear, however, how such different envelope temperatures could be reached at very similar accretion rates. The composition of the accreted material is not expected to change much, since fresh matter arrives at high rates.

The situation at high mass accretion rates becomes even more confusing when one considers the bright "GX atoll" sources (GX 3+1, GX 13+1, GX 9+1, GX 9+9) as well as the other Z sources (Sco X-1, GX 5-1, GX 340+0, GX 349+2, Cyg X-2). The former are thought to accrete with rates around $10 \%$ of the Eddington mass accretion rate (Psaltis \& Lamb 1998; see also Kuulkers \& van der Klis 2000). For those sources, only short $(\simeq 10-$ $15 \mathrm{~s}$ duration) bursts are seen (infrequently) in GX $3+1$ (Makishima et al. 1983; Asai et al. 1993; Molkov et al. 1999; Kuulkers \& van der Klis 2000) and GX 13+1 (Matsuba et al. 1995) and no bursts have been reported for GX 9+1 and GX 9+9, despite ample observing times.

The X-ray spectral and fast timing properties of the other five canonical $\mathrm{Z}$ sources are very similar to those of GX 17+2 (see Hasinger \& van der Klis 1989; van der Klis 2000), and thus one would infer comparable mass accretion rates. Of the five of these sources, however, only Cyg X-2 shows X-ray bursts, which are typical He flashes, whereas the others do not burst at all (despite ample observing times). Since at very high accretion rates no bursts are expected one would then naively infer that the average mass accretion rate $i s$ different, i.e. higher, in the four non-bursting $\mathrm{Z}$ sources with respect to the two bursting 
Z sources. But this would imply that the correlated X-ray spectral and fast timing properties are not a function of the inferred mass accretion rate; van der Klis (2001) has recently proposed a way in which the correlated properties could vary in response to changes in the mass accretion rate without being a function of it. A slightly different suggestion was made by Homan et al. (2001) who propose that the mass accretion rate is not the parameter that determines the position along the $\mathrm{Z}$ track. As a consequense, bursts that occur at a similar position along the $\mathrm{Z}$ might occur at different mass accretion rates and therefore have different proporties. Note also that differences in mass accretion rate per unit area may be of importance here, as was already suggested by Kuulkers et al. (1997) to explain the properties of quasi-periodic oscillations occurring on the $\mathrm{HB}$ and upper parts of the NB, and the presence of bursts in GX $17+2$. On the other hand, since the envelope temperatures and composition also influence the bursting properties, they may be of importance too (e.g. Taam et al. 1996). Future modelling may provide more insight in the processes involved.

\section{Conclusions}

- We found ten X-ray bursts in all data on GX 17+2 obtained with the RXTE/PCA to date. Three of them were short $(\simeq 10 \mathrm{~s})$, while the others lasted from $\simeq 6$ to $25 \mathrm{~min}$. All the bursts showed spectral softening during the decay, indicative of cooling of the neutron star. No evidence for high-frequency $(>100 \mathrm{~Hz})$ oscillations at any phase of the bursts is seen. We find no evidence for correlations of the burst properties with respect to the source position in the colour-colour diagram (presumably a function of the mass accretion rate). The long bursts are consistent with being due to mixed $\mathrm{H} / \mathrm{He}$ burning, triggered by thermally unstable He ignition, as expected at the inferred near-Eddington mass accretion rates in GX $17+2$. However, the presence of short bursts in GX $17+2$, as well as short bursts in CygX-2, another persistently bright LMXB, and no bursts in the other four similar LMXBs Sco X-1, GX 5-1, GX 340+0 and GX 349+2, is not accounted for in the current X-ray burst theories. Note that this also holds for the bright "GX atoll" sources GX $3+1$, GX $13+1$, GX $9+1$ and GX 9+9, which are thought to accrete near one tenth of the Eddington rate. Of these four atoll sources, only GX $3+1$ and GX $13+1$ infrequently show short $(\simeq 10-15 \mathrm{~s})$ X-ray bursts, whereas GX $9+1$ and GX $9+9$ have not been seen to burst at all.

- We found that two-component spectral fits to the total burst emission, as has been suggested previously, do not give satisfactory results whenever the persistent emission before the burst contains a black-body component. On the other hand, the "standard" spectral fit analysis, in which the burst emission is modeled by black-body radiation after subtraction of the persistent emission before the burst, does provide satisfactory results. This means that whenever there is a black-body contribution in the persistent emission before the burst, it is probably also present during the burst itself (i.e. the burst black-body emission appears on top of the persistent black-body emission). This implies that the burst emission does not arise at the same site as the black-body component often seen in the persistent emission. We find evidence that the emission region of the persistent black-body component is larger than that of the burst emission, which indicates that it probably originates in an expanded boundary layer, as recent modeling indicates.

- Five of the long bursts showed evidence for an expanding photosphere during the first seconds of the burst, presumably due to the burst luminosity reaching the Eddington limit. The contraction phase differs in duration between the bursts, the longest being $\simeq 3$ min. The net burst fluxes reached during the radius expansion/contraction phase are all more or less similar, which is indicative of the Eddington limit as observed at earth.

- The persistent flux just before the burst is inferred to be up to a factor 2 higher than the net burst flux during the radius expansion/contraction phase. If in both cases the emission is isotropic, this implies that the persistent emission is up to a factor of 2 higher than the Eddington luminosity. We suggest that the burst emission is (highly) anisotropic. Since both the persistent luminosity in GX 17+2 and the net burst luminosity during the expansion/contraction phase are thought to have near Eddington values, we then derive an anisotropy factor of $\sim 2$ for the burst emission.

- When the burst luminosity is near Eddington values, deviations from pure black-body radiation are evident below $10 \mathrm{keV}$. Similar deviations have been seen during (long) X-ray bursts in other low-mass X-ray binaries, and can not be explained by spectral hardening. Assuming that the black-body approximation is, nevertheless valid, that the net burst peak fluxes during the radius expansion/contraction phase equals the Eddington limit as seen on Earth, and using an anisotropy factor of 2, we estimate the distance to GX $17+2$, taking into account gravitational redshift effects and spectral hardening. For "standard" parameters $\left(M_{\mathrm{ns}}=1.4 M_{\odot}\right.$, cosmic composition) we derive a distance $\sim 8 \mathrm{kpc}$, with a systematic uncertainty of up to $\sim 30 \%$.

Acknowledgements. We thank Lars Bildsten, Jean in 't Zand and Marten van Kerkwijk for providing comments on earlier drafts of this paper, Keith Jahoda for providing up-to-date response matrices, and the referee for carefully reading the manuscript. EK thanks the MIT/RXTE X-ray burst group for making us aware of the burst-like event $\mathrm{f} 1$, which resulted in our detection of the events f2-f4. This work was supported in part by the Netherlands Organization for Scientific Research (NWO). WHGL is grateful for support from NASA.

\section{Appendix A: X-ray bursts from GX $17+2$ observed with other instruments}

X-ray bursts from GX $17+2$ have been previously seen with the Monitor Proportional Counter (MPC) onboard the Einstein Observatory (Kahn \& Grindlay 1984), the 
second Fine Modulation Collimator (FMC-2) onboard Hakucho (Tawara et al. 1984c) and the Medium Energy (ME) experiment onboard EXOSAT (Sztajno et al. 1986; Kuulkers et al. 1997). Not all the bursts parameters as tabulated in Table 4 for the bursts seen with the RXTE/PCA have been given by these authors. In order to compare our bursts in more detail with the other observed bursts we calculated these parameters from the information given in those papers. Since the X-ray energy ranges in which the different instruments operate are comparable, and most of the (burst) emission is radiated in these energy bands, such a comparison is feasible. In the next subsections we describe how the parameters were determined for the different bursts.

\section{A.1. MPC/Einstein}

Kahn \& Grindlay (1984, see also Kahn et al. 1981) observed a burst from GX $17+2$ in 1980 with the MPC onboard Einstein, with a decay time of $\simeq 7.5 \mathrm{~s}$. From their background subtracted count rate profiles in the 1.4 $14.4 \mathrm{keV}$ energy band (their Fig. 1) we determined the values for $\gamma$ and $\tau$. The value of $\gamma$ is consistent with what is inferred using the quoted net peak and persistent count rate of 0.15 and $0.56 \mathrm{Crab}(2-6 \mathrm{keV})$, respectively, by Kahn et al. (1981). The persistent flux before the burst was given as $1.56 \pm 0.02 \times 10^{-8} \mathrm{erg} \mathrm{cm}^{-2} \mathrm{~s}^{-1}$ (1-20 keV). Kahn \& Grindlay (1984) report a maximum peak flux of $9.1 \times 10^{-8} \mathrm{erg} \mathrm{cm}^{-2} \mathrm{~s}^{-1}(1-20 \mathrm{keV})$. No error is given, but only a \pm -sign. It is not clear whether this is a typo, or if this means that the value was approximate. Their peak flux is much higher than observed for the other bursts. An averaged net spectrum over the burst profile (first $7.68 \mathrm{~s}$ ) was modeled as black-body emission. Values of $3.8 \lesssim T_{\mathrm{bb}} \lesssim 9.3 \mathrm{keV}$ and black-body emission area, $A_{\mathrm{bb}}$, of $0.56 \lesssim A_{\mathrm{bb}} \lesssim 3.2 \times 10^{12} \mathrm{~cm}^{2}$ (at $10 \mathrm{kpc}$ ) were found. This, in principle, may give us a handle on the observed fluence. However, this gives us a value of $E_{\mathrm{b}}$ of $\simeq 161 \times 10^{-8} \mathrm{erg} \mathrm{cm}^{-2}$ (using $T_{\mathrm{bb}} \simeq 6.55 \mathrm{keV}$ and $\mathrm{A}_{\mathrm{bb}} \simeq 1.88 \times 10^{12} \mathrm{~cm}^{2}$ ), which is also much larger than observed for the other short bursts. We, therefore, do not quote the peak flux and burst fluence in Table A.1. Using the Einstein database maintained at HEASARC ${ }^{4}$ we found the start of the (simultaneous HRI) observation as being March 29, 1980, 00:48:06 (UTC). The burst was observed $\simeq 57$ min later (Kahn et al. 1981). Using this and the observed rate just before the burst we determined an upper limit on $\alpha$.

\section{A.2. FMC-2/Hakucho}

Four bursts (two in 1981 and two in 1982; denoted AD) were found, originating from GX $17+2$, during pointed observations with the FMC-2 onboard Hakucho (Tawara et al. 1984c). They had e-folding decay times of $\simeq 100$ s (B, C) or $\simeq 300 \mathrm{~s} \mathrm{(A,} \mathrm{D).} \mathrm{For} \mathrm{each} \mathrm{burst} \mathrm{Tawara} \mathrm{et} \mathrm{al.} \mathrm{(1984c)}$

\footnotetext{
${ }^{4}$ See http://heasarc.gsfc.nasa.gov/
}

tabulated the net peak, net total (=fluence) and persistent flux in FMC-2 $\mathrm{cts} \mathrm{s}^{-1}$ in the $1-22 \mathrm{keV}$ band. From this they calculated $\gamma$. Using this information we calculated $\tau$ and verified $\gamma$. Tawara et al. (1984c) also provided the total integrated energy fluxes (= total fluence) of the two bursts in 1981 and the two bursts in 1982, together with the integrated persistent fluxes over $40 \mathrm{hr}$ and $50 \mathrm{hr}$, respectively. Note that it is not clear if the fluence and persistent flux are both given for the $1-22 \mathrm{keV}$ energy band, and whether the persistent fluxes are corrected for interstellar absorption or not. From this it was estimated that $\alpha$ was of the order of 1000. Since the net fluence in counts of all bursts individually are given, we can determine the individual fluence in energy flux. Also, the (mean) persistent flux during the two observations can be determined. Moreover, assuming that between bursts A and B, which occurred $12.5 \mathrm{hr}$ after each other, no other bursts occur, we give a more exact value for $\alpha$ in this case (assuming a constant persistent flux). For the others we just assume the given value of 1000 . We also give an estimate of the net peak burst flux, from the observed values of $F_{\text {pers }}$ and $\gamma$.

\section{A.3. ME/EXOSAT}

Two bursts were found in the EXOSAT/ME observations taken in 1984 and 1985 with durations of $\simeq 10 \mathrm{~s}$ and $>5 \mathrm{~min}$ (Sztajno et al. 1986; denoted as burst I and II by Kuulkers et al. 1997). The persistent flux $(2-20 \mathrm{keV})$ before a burst was determined from the spectral fits done by Sztajno et al. (1986), who used a bremsstrahlung plus a black-body component, subjected to interstellar absorption. The values for the bremsstrahlung parameters and $N_{\mathrm{H}}$ were given in their text, whereas the black-body results were indicated in their Figs. 4 and 5. The spectral fit results to the net burst emission are shown in their Figs. 2 and 3, from which we determined the bolometric maximum net peak flux and the fluence. To determine the "rest" fluence, we used the same approach as mentioned in Sect. 3.2.5, and fitted an exponential to the burst flux decay. In the case of burst I we have only 3 values; we therefore assumed that at infinity the burst flux decays to zero, while the exponential start time was set to the start of the burst. Since the spectral time resolution is not sufficient, we determined the burst parameters $\gamma$ and $\tau$ from the observed raw (i.e. not dead-time corrected) count rate profiles (1-20 keV) given in their Fig. 1. We corrected the light curves for the "variable" dead time (van der Klis 1989), as appropriate for the EXOSAT High Energy Resolution (HER) modes (see e.g. Kuulkers 1995). Sztajno et al. (1986) noted that in both bursts the maximum flux is $\simeq 40 \%$ above the persistent level, indicating $\gamma \simeq 2.5$, in rough agreement with our estimates. Using the start time of the two observations during which the bursts occurred as given by Kuulkers et al. (1997), and assuming that the persistent flux was constant, we were able to determine lower limits on $\alpha$. Note that Sztajno et al. (1986) gave rough upper limits of 
Table A.1. Burst parameters of other bursts in the literature.

\begin{tabular}{|c|c|c|c|c|c|c|}
\hline burst & $F_{\text {pers }}{ }^{a}$ & $F_{\mathrm{bb}, \max }{ }^{b}$ & $E_{\mathrm{b}}{ }^{c}$ & $\tau(\mathrm{s})$ & $\gamma$ & $\alpha$ \\
\hline \multicolumn{7}{|c|}{ Kahn \& Grindlay (1984) } \\
\hline & $1.56 \pm 0.02$ & see text & see text & $6.7 \pm 1.4$ & $3.4 \pm 0.2$ & $\gtrsim 1750$ \\
\hline \multicolumn{7}{|c|}{ Tawara et al. (1984c) } \\
\hline A & $\simeq 2.8$ & $\simeq 1.5$ & $\simeq 346$ & $367 \pm 43$ & $1.83 \pm 0.19$ & $\sim 1000$ \\
\hline B & $\simeq 2.8$ & $\simeq 2.0$ & $\simeq 94$ & $75 \pm 8$ & $1.38 \pm 0.13$ & $\simeq 1300$ \\
\hline $\mathrm{C}$ & $\simeq 2.9$ & $\simeq 1.7$ & $\simeq 114$ & $109 \pm 37$ & $1.77 \pm 0.20$ & $\sim 1000$ \\
\hline $\mathrm{D}$ & $\simeq 2.9$ & $\simeq 2.1$ & $\simeq 406$ & $309 \pm 27$ & $1.41 \pm 0.11$ & $\sim 1000$ \\
\hline \multicolumn{7}{|c|}{ Sztajno et al. (1986) } \\
\hline I & $1.51 \pm 0.13$ & $1.1 \pm 0.4$ & $4.7 \pm 1.0$ & $6.27 \pm 1.2$ & $3.6 \pm 0.2$ & $\gtrsim 1450$ \\
\hline II & $1.86 \pm 0.15$ & $0.99 \pm 0.05$ & $114 \pm 3$ & $132 \pm 4$ & $2.13 \pm 0.02$ & $\gtrsim 650$ \\
\hline \multicolumn{7}{|c|}{ Kuulkers et al. (1997) } \\
\hline III & - & - & - & $\simeq 27$ & $\simeq 2.7$ & $\gtrsim 7000$ \\
\hline IV & - & - & - & $\simeq 100$ & $\simeq 3.2$ & $\simeq 2200$ \\
\hline
\end{tabular}

$\sim 10000$ and $\sim 500$, for burst I and II, respectively, which are more or less consistent with our determined values.

For the two bursts (denoted as burst III and IV) observed in 1986 discussed by Kuulkers et al. (1997) we only estimated $\gamma$ and $\tau$ from the observed (dead-time and background corrected) count rate profiles in the $1-20 \mathrm{keV}$ band as displayed in their figure 7. $\tau$ is determined using the integrated net burst count rate profile and peak count rate. No spectral information is available for the persistent or burst emission in order to determine $F_{\mathrm{bb}, \max }, E_{\mathrm{b}}$ and $\alpha$, although they note that $\alpha$ is probably larger than $\sim 1000$ for the second burst, if no bursts occurred in between the two bursts. However, if we naively assume that the persistent count rate is constant between bursts, and that between the start of the 1986 EXOSAT observation and the first burst, and between the two bursts, no other burst occurred, we can still get a crude estimate of (the lower limit on) the value of $\alpha$.

\section{Appendix B: Distance estimate and gravitational redshift effect}

Most of the distances derived from radius expansion bursts in the literature are solely based on the observed fluxes at maximum expansion, $F_{\mathrm{bb} \text {, } a x}$, and assuming this equals the (non-relativistic) Eddington luminosity, $L_{\text {Edd,non_rel, }}$ from a source at distance $d$. Here one uses $L_{\text {Edd,non_rel }}=$ $4 \pi c G M_{\mathrm{ns}} / \kappa$, where $c$ is the speed of light, $G$ the gravitational constant, $M_{\mathrm{ns}}$ the mass of the neutron star and $\kappa$ the electron scattering opacity. Then the distance may be estimated as:

$d=\left(\frac{L_{\mathrm{Edd}, \text { non_rel }}}{4 \pi \xi F_{\mathrm{bb}, \max }}\right)^{1 / 2}$,

where $\xi$ is the anisotropy factor (for isotropic radiation $\xi=$ 1). However, when the photosphere remains rather close to the neutron star, gravitational redshift effects become important (due to relativistic time dilation, see e.g. Lewin et al. 1993). The distance derived using Eq. (B.1) is in fact only valid for values of the photosphere radius, $R \gg$ $R_{\mathrm{ns}}$ (see below). For $R \gtrsim R_{\mathrm{ns}}$ Eq. (B.1) in principle only provides an upper limit on $d$.

The (relativistic) Eddington luminosity for an observer near the neutron star is given by:

$L_{\mathrm{Edd}}=\left(4 \pi c G M_{\mathrm{ns}} / \kappa\right)\left[1-2 G M_{\mathrm{ns}} /\left(R c^{2}\right)\right]^{-1 / 2}$.

For an observer at Earth, Eq. (B.2) becomes, taking into gravitational redshift effects:

$L_{\mathrm{Edd}, \infty}=L_{\mathrm{Edd}}\left[1-2 G M_{\mathrm{ns}} /\left(R c^{2}\right)\right]=L_{\mathrm{Edd}}\left[1-R_{\mathrm{g}} / R\right]$,

where $R_{\mathrm{g}}$ is the Schwarzschild radius $\left(R \geq 1.5 R_{\mathrm{g}}\right.$; see Lewin et al. 1993). Also the effective black-body temperature measured at Earth, $T_{\text {eff, } \infty}$, is affected by the gravitational potential:

$T_{\text {eff }, \infty}=T_{\text {eff }}\left[1-R_{\mathrm{g}} / R\right]^{1 / 2}$,

where $T_{\text {eff }}$ is the effective black-body temperature near the neutron star. Assuming that the source radiates as a black-body, we know that:

$L_{\mathrm{Edd}}=4 \pi R^{2} \sigma T_{\mathrm{eff}}^{4}$.

For $L_{\mathrm{Edd}, \infty}$ we also have:

$L_{\mathrm{Edd}, \infty}=4 \pi d^{2} \xi F_{\mathrm{Edd}, \infty}$,

where $F_{\mathrm{Edd}, \infty}$ is the Eddington flux received at Earth.

Equations (B.3) and (B.4) are strictly valid only for non-rotating stars, but it is still approximately correct for stars with rotation periods of a few milliseconds. $F_{\mathrm{Edd}, \infty}$ is set equal to the observed net-burst fluxes, $F_{\mathrm{bb} \text {,net }}$, during the radius expansion/contraction phase. As noticed in 
Table B.1. Distance $(d$ in $\mathrm{kpc})$ and radius at touch down from radius expansion bursts ${ }^{a}$.

\begin{tabular}{|c|c|c|c|c|c|c|c|}
\hline \multirow[b]{2}{*}{ burst } & \multicolumn{3}{|c|}{$0.25 \mathrm{~s}$ spectra } & \multicolumn{3}{|c|}{$16 \mathrm{~s}$ spectra } & \multirow{2}{*}{$\begin{array}{l}R_{\mathrm{td}}^{b} \\
(\mathrm{~km})\end{array}$} \\
\hline & $\#^{c}$ & $d_{\text {non_rel }} d$ & $d_{\mathrm{rel}}{ }^{e}$ & \# & $d_{\text {non_rel }}$ & $d_{\text {rel }}$ & \\
\hline $\mathrm{b} 4$ & 84 & $11.4 \pm 0.3$ & $10.8 \pm 0.3$ & 4 & $11.6 \pm 0.3$ & $10.9 \pm 0.3$ & 18.3 \\
\hline b6 & 83 & $12.1 \pm 0.5$ & $11.4 \pm 0.5$ & 6 & $12.2 \pm 0.1$ & $11.1 \pm 0.1$ & 12.8 \\
\hline b7 & 94 & $12.9 \pm 0.6$ & $12.0 \pm 0.5$ & 2 & $12.7 \pm 0.2$ & $11.8 \pm 0.1$ & 16.7 \\
\hline b8 & 93 & $12.3 \pm 0.6$ & $11.5 \pm 0.5$ & 2 & $12.1 \pm 0.1$ & $11.2 \pm 0.1$ & 14.4 \\
\hline b9 & 82 & $12.4 \pm 0.5$ & $11.5 \pm 0.5$ & 1 & 12.1 & 11.1 & 14.9 \\
\hline \multicolumn{2}{|c|}{ Average: } & $12.2 \pm 0.2$ & $11.4 \pm 0.2$ & & $12.1 \pm 0.1$ & $11.2 \pm 0.1$ & $15.4 \pm 1.0$ \\
\hline
\end{tabular}

\footnotetext{
${ }^{a}$ For the parameters used we refer to the text.

${ }^{b}$ Black-body radius $R$ at touch down for a local observer (see text).

${ }^{c}$ Number of spectra in the expansion/contraction phase.

${ }^{d}$ Not taking into account gravitational redshift effects.

${ }^{e}$ Taking into account gravitational redshift effects.
}

Sect. 5.2, it is not easy to infer $T_{\text {eff, } \infty}$ from our observed values of $T_{\mathrm{bb}}$. We therefore fixed the hardening factor, $T_{\mathrm{bb}} / T_{\text {eff, } \infty}$, to different values in order to assess its effect. We also assume certain values for $M_{\mathrm{ns}}$ and $\xi$, and are then left with five equations (B.2-B.6) and five unknown variables ( $L_{\mathrm{Edd}}, L_{\mathrm{Edd}, \infty}, T_{\mathrm{eff}}, R$ and $d$ ); this enables us to derive a distance estimate. First, one can eliminate $L_{\text {Edd }}$ and $T_{\text {eff }}$ by combining Eqs. (B.2), (B.4) and (B.5), and one gets the following equality (for $R \geq 1.5 R_{\mathrm{g}}$ ):

$$
\frac{c G M_{\mathrm{ns}}}{\kappa \sigma \mathrm{T}_{\mathrm{eff}, \infty}^{4}}=R^{2}\left(\frac{R}{R-R_{\mathrm{g}}}\right)^{\frac{3}{2}}
$$

Since this equation cannot be solved easily in an analytic way, we solved for $R$ numerically. Using $R$ we can then determine $L_{\mathrm{Edd}, \infty}$ (Eq. (B.3)), which in turn can be used to determine the distance, $d$ (Eq. (B.6)).

In the low-temperature limit the electron scattering opacity, $\kappa$, is given by $\kappa=0.2(1+X) \mathrm{cm}^{2} \mathrm{~g}^{-1}$, where $X$ is the hydrogen fraction (by mass) of the photospheric matter (see e.g. Lewin et al. 1993). Note that for cosmic compositions $X=0.73$. At very high temperatures (i.e. probably near the peak of bursts), however, the scattering electrons become relativistic and $\kappa$ may instead be approximated by (in the low-density limit) $\kappa=\kappa_{0}\left[1+(k T / 39.2 \mathrm{keV})^{0.86}\right]^{-1}$, where $\kappa_{0}$ is same as for the low-temperature limit (Paczyński 1983). It is not entirely clear how $k T$ relates to our observed values of $k T_{\mathrm{bb}}$, but one may assume $k T=k T_{\mathrm{bb}}$ (e.g. van Paradijs et al. 1990). We use the high temperature electron scattering opacity in our calculations. The estimated distances differ by a factor of $\simeq 1.05$ with respect to the estimates when taking the electron scattering opacity in the lowtemperature limit (see also Table B.2).

The above described procedure for estimating the distance was followed using the values for $F_{\mathrm{bb} \text {,net }}$ and $T_{\mathrm{bb}}$ for each of the spectral fits to the $0.25 \mathrm{~s}$ and $16 \mathrm{~s}$ spectra in the radius expansion/contraction phase. When we assume no spectral hardening, i.e. $T_{\mathrm{eff}, \infty}=T_{\mathrm{bb}}$, and "standard" parameters $\left(M_{\mathrm{ns}}=1.4 M_{\odot}, X=0.73\right)$ no solutions can be found for those spectra having $k T_{\mathrm{bb}} \gtrsim 1.75 \mathrm{keV}$, i.e. only at the largest expansion radii do we have solutions (see also Table B.2) ) $^{5}$ The highest observed values of $k T_{\mathrm{bb}}$ during the radius expansion/contraction phase give the strongest constraints on the spectral hardening factor. This is at the moment of "touch-down", i.e. at the vertex of the horizontal track and diagonal track in the flux versus temperature diagram. This locus is well defined for our long bursts observed in GX $17+2$. Using the loci of the radius expansion bursts $\mathrm{b} 4, \mathrm{~b} 6-\mathrm{b} 9$, we find that the minimum value of the (constant) hardening factor is between 1.34 (burst b4) and 1.52 (burst b6). The distance and radius $R$ as inferred at the loci both increase for increasing spectral hardening (e.g. for burst b6, the distance increases by a factor $\simeq 1.1$, while $R$ increases by a factor of $\simeq 2$ when the spectral hardening factor increases from 1.6 to 2$)$.

In Table B.1 the results for "standard" burst parameters can be seen, i.e. $M_{\mathrm{ns}}=1.4 M_{\odot}$, cosmic composition of the photospheric matter, isotropic radiation $(\xi=1)$, as well as taking $T_{\mathrm{bb}} / T_{\text {eff, } \infty}=1.7$ and using the electron scattering opacity appropriate for high temperatures. We show the distance as derived by including gravitational redshift effects $\left(d_{\text {rel }}\right)$ and not including these effects $\left(d_{\text {non_rel }}\right.$, i.e. using Eq. (B.1)). The errors are set equal to the observed variances in the derived distances.

By relaxing the assumptions of standard burst parameters (i.e. varying $M_{\mathrm{ns}}, X$ and $\xi$ individually within reasonable limits, while keeping the others at the "standard" values) one can get an estimate of the systematic uncertainties involved (see e.g. also Kuulkers \& van der Klis 2000). The results are shown in Table B.2 for burst b6, which is the longest burst of our sample. We show the results assuming a neutron star mass of $M_{\mathrm{ns}}=2 M_{\odot}$,

\footnotetext{
${ }^{5}$ Note that the gravitational redshift corrected distance estimates by Smale (1998: Cyg X-2), Kuulkers \& van Klis (2000: GX 3+1) and Kaptein et al. (2000: 1RXS J171824.2-402934) are derived using $T_{\mathrm{eff}, \infty}=T_{\mathrm{bb}}$ and $R=R_{\mathrm{bb}}$, thus only using Eqs. (B.2), (B.3) and (B.6).
} 
Table B.2. Distance ( $d$ in kpc) and radius at touch down for burst b6 using different assumptions ${ }^{a}$.

\begin{tabular}{|c|c|c|c|c|c|}
\hline \multirow[b]{2}{*}{ parameter $^{a}$} & \multicolumn{2}{|c|}{$0.25 \mathrm{~s}$ spectra } & \multicolumn{2}{|c|}{16 s spectra } & \multirow{2}{*}{$\begin{array}{l}R_{\mathrm{td}}{ }^{b} \\
(\mathrm{~km})\end{array}$} \\
\hline & $d_{\text {non_rel }}^{c}$ & $d_{\text {rel }}^{d}$ & $d_{\text {non_rel }}$ & $d_{\text {rel }}$ & \\
\hline standard $^{e}$ & $12.1 \pm 0.5$ & $11.4 \pm 0.5$ & $12.2 \pm 0.1$ & $11.1 \pm 0.1$ & 12.8 \\
\hline$M_{\mathrm{ns}}=2.0 M_{\odot}$ & $14.4 \pm 0.6$ & $13.5 \pm 0.6$ & $14.6 \pm 0.1$ & $12.7 \pm 0.2$ & 13.0 \\
\hline$X=0$ & $15.9 \pm 0.7$ & $15.3 \pm 0.6$ & $16.1 \pm 0.1$ & $15.1 \pm 0.1$ & 18.9 \\
\hline low- $T \kappa$ & $11.6 \pm 0.5$ & $11.0 \pm 0.5$ & $11.7 \pm 0.1$ & $10.6 \pm 0.1$ & 11.8 \\
\hline$\xi=2$ & $8.5 \pm 0.3$ & $8.1 \pm 0.3$ & $8.6 \pm 0.1$ & $7.9 \pm 0.1$ & 12.8 \\
\hline$\xi=0.5$ & $17.1 \pm 0.7$ & $16.2 \pm 0.7$ & $17.3 \pm 0.1$ & $15.8 \pm 0.1$ & 12.8 \\
\hline$T_{\mathrm{bb}} / T_{\mathrm{eff}, \infty}=2$ & $12.1 \pm 0.5$ & $11.7 \pm 0.5$ & $12.2 \pm 0.1$ & $11.6 \pm 0.1$ & 19.9 \\
\hline$T_{\mathrm{bb}} / T_{\mathrm{eff}, \infty}=1^{f}$ & $12.1 \pm 0.5(83)$ & $10.5 \pm 0.7(9)$ & $12.2 \pm 0.1(6)$ & - & - \\
\hline \multicolumn{6}{|c|}{${ }^{a}$ See text for details. } \\
\hline \multicolumn{6}{|c|}{${ }^{b}$ Black-body radius $R$ at touch down for a local observer (see text). } \\
\hline \multicolumn{6}{|c|}{${ }^{c}$ Not taking into account gravitational redshift effects. } \\
\hline \multicolumn{6}{|c|}{${ }^{d}$ Taking into account gravitational redshift effects. } \\
\hline \multicolumn{6}{|c|}{${ }^{e} M_{\mathrm{ns}}=1.4 M_{\odot}, X=0.73, T_{\mathrm{bb}} / T_{\mathrm{eff}, \infty}=1.7$, high- $T \kappa, \xi=1}$. \\
\hline$f=3$ & is the number of & 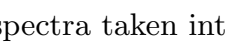 & & & \\
\hline
\end{tabular}

in the range required in models for explaining the $\mathrm{kHz}$ QPO in neutron star LMXBs (see e.g. Stella et al. 1999; Stella 2001; Lamb \& Miller 2001), and close to the dynamical neutron mass estimate of Cyg X-2 using optical spectroscopy/photometry, $M_{\mathrm{ns}}=1.78 \pm 0.23$ (Orosz \& Kuulkers 1999). Note that the distance derived is rather high (Table B.2). We also made the assumption that the photospheric composition is hydrogen-poor (i.e. $X=0$ ), as has been argued to be the case during radius expansion (see Sugimoto et al. 1984). However, the contraction phase of long bursts are typical of unstable mixed hydrogen/helium burning (see Sect. 5.4), which suggests that this is not a reasonable assumption. Also, the persistent mass accretion rate is inferred to be high, making it plausible that the photosphere will be continuously supplied with new hydrogen matter. Moreover, the distances derived are rather high (see Table B.2). We changed the anisotropy factor between the reasonable values $0.5 \lesssim \xi \lesssim 2$ (see van Paradijs \& Lewin 1987; Lewin et al. 1993). Finally, we compared the distance values derived using values for $\kappa$ valid for low temperatures. The results shown in Table B.2 suggests that the systematic uncertainties on the derived distance are up to $\sim 30 \%$.

\section{References}

Asai, K., Dotani, T., Nagase, F., et al. 1993, PASJ, 45, 801 Babul, A., \& Paczyński, B. 1987, ApJ, 323, 582

Bildsten, L. 1998, in The Many Faces of Neutron Stars, ed. R. Buccheri, J. van Paradijs, \& M. A. Alpar (Dordrecht: Kluwer), 419

Bildsten, L. 2000, in Cosmic Explosions, ed. S. S. Holt, \& W. W. Zhang, AIP, 522, 359

Bradt, H. V., Rothschild, R. E., \& Swank, J. H. 1993, A\&AS, 97,355

Chevalier, C., \& Ilovaisky, S. A. 1990, A\&A, 228, 115

Christian, D., \& Swank, J. H. 1997, ApJS, 197, 177

Church, M. J., \& Bałucińska-Church, M. 2001, A\&A, 369, 915
Cornelisse, R., Heise, J., Kuulkers, E., Verbunt, F., \& in 't Zand, J. J. M. 2000, A\&A, 357, L21

Cornelisse, R., Kuulkers, E., in 't Zand, J. J. M., Verbunt, F., \& Heise, J. 2002, A\&A, 382, 174

Di Salvo, T., Stella, L., Robba, N. R., et al. 2000, ApJ, 544, L119

Ebisuzaki, T. 1987, PASJ, 39, 287

Fortner, B., Lamb, F. K., \& Miller, G. S. 1989, Nature, 342, 775

Fujimoto, M. Y., Hanawa, T., \& Miyaji, S. 1981, ApJ, 247, 267

Fujimoto, M. Y., Sztajno, M., Lewin, W. H. G., \& van Paradijs, J. 1988, A\&A, 199, L9

Fushiki, I., \& Lamb, D. Q. 1987, ApJ, 323, L55

Ghosh, P., \& Lamb, F. K. 1992, in Proc. NATO Conf. on X-ray Binaries and Reycled Pulsars, ed. E. P. J. van den Heuvel, \& S. A. Rappaport (Kluwer, Dordrecht), 487

Grindlay, J., \& Heise, J. 1975, IAUC, 2879

Grindlay, J., Gursky, H., Schnopper, H., et al. 1975, ApJ, 205, L127

Groth, E. J. 1975, ApJS, 29, 285

Hasinger, G. 1987, A\&A, 186, 153

Hasinger, G., \& van der Klis, M. 1989, A\&A, 225, 79

Hasinger, G., van der Klis, M., Ebisawa, K., Dotani, T., \& Mitsuda, K. 1990, A\&A, 235, 131

Hoffman, J. A., Lewin, W. H. G., Doty, J., et al. 1978b, ApJ, 221, L57

Hoffman, J. A., Marshall, H. L., \& Lewin, W. H. G. 1978a, Nature, 271, 630

Homan, J., van der Klis, M., Jonker, P. G., et al. 2001, ApJ, in press [astro-ph/0104323]

Kahn, S. M., \& Grindlay, J. E. 1984, ApJ, 281, 826

Kahn, S., Grindlay, J., Halpern, J., \& Ladd, E. 1981, IAU Circ., 3625

Kaptein, R. G., in 't Zand, J. J. M., Kuulkers, E., et al. 2000, A\&A, 358, L71

Koshut, T. M., Paciesas, W. S., Kouveliotou, C., et al. 1996, ApJ, 463, 570

Kuulkers, E. 1995, Ph.D. Thesis, University of Amsterdam

Kuulkers, E. 2001, ATel \#68

Kuulkers, E., \& van der Klis, M. 2000, A\&A, 356, L45 
Kuulkers, E., van der Klis, M., \& van Paradijs, J. 1995, ApJ, 450, 748

Kuulkers, E., van der Klis, M., Oosterbroek, T., van Paradijs, J., \& Lewin, W. H. G. 1997, MNRAS, 287, 495

Kuulkers, E., in 't Zand, J. J. M., van Kerkwijk, M. H., et al. 2002, A\&A, 382, 503

Lamb, D. Q. 2000, ApJS, 127, 395

Lamb, F. K. 1989, in Two Topics in X-ray Astronomy, 23rd ESLAB Symposium, ed. J. Hunt, \& B. Battrick, ESA SP-296, 215

Lamb, F. K., \& Miller, M. C. 2001, ApJ, 554, 1210

Leahy, D. A., Darbro, W., Elsner, R. F., et al. 1983, ApJ, 266, 160

Lewin, W. H. G., Vacca, W. D., \& Basinska, E. M. 1984, ApJ, 277, L57

Lewin, W. H. G., van Paradijs, J., \& Taam, R. E. 1993, Space Sci. Rev., 62, 223

London, R. A., Taam, R. E., \& Howard, W. M. 1984, ApJ, 287, L27

London, R. A., Taam, R. E., \& Howard, W. M. 1986, ApJ, 306,170

Madej, J. 1997, A\&A, 320, 177

Makishima, K., Mitsuda, K., Inoue, H., et al. 1983, ApJ, 267, 310

Maraschi, L., \& Cavaliere, A. 1977, HiA, 4, 127

Marshall, H. L. 1982, ApJ, 260, 815

Matsuba, E., Dotani, T., Mitsuda, K., et al. 1995, PASJ, 47, 575

Molkov, S. V., Grebenev, S. A., Pavlinsky, M. N., \& Sunyaev, R. A. 1999, in Proc. of the 3rd INTEGRAL Workshop The Extreme Universe, ApLC, 38, 141

Muno, M. P., Fox, D. W., Morgan, E. H., \& Bildsten, L. 2000, ApJ, 542, 1016

Orosz, J. A., \& Kuulkers, E. 1999, MNRAS, 305, 132

Paczyński, B. 1983, ApJ, 267, 315

Penninx, W., Damen, E., van Paradijs, J., Tan, J., \& Lewin, W. H. G. 1989, A\&A, 208, 146

Popham, R., \& Sunyaev, R. 2001, ApJ, 547, 355

Psaltis, D., \& Lamb, F. K. 1998, in Neutron Stars and Pulsars, ed. N. Shibazaki, N. Kawai, S. Shibata, \& T. Kifune, Tokyo, Universal Academy, 179

Schatz, H., Bildsten, L., Cumming, A., \& Wiescher, M. 1999, ApJ, 524, 1014

Schulz, N. S. 1999, ApJ, 511, 304

Smale, A. 1998, ApJ, 498, L141

Smale, A. P. 2001, ApJ, in press [astro-ph/0107354]

Stella, L. 2001, in Proceedings X-ray Astronomy '99 Stellar Endpoints, AGN and the Diffuse Background, ed. G. Malaguti, G. Palumbo, \& N. White, AIP Series of
Conference Proceedings, in press [astro-ph/0011395]

Stella, L., Vietri, M., \& Morsink, M. 1999, ApJ, 524, L63

Strohmayer, T. 1998, Nucl. Phys. B. (Proc. Suppl.) 69/1-3, 129

Strohmayer, T. 2001, in Proceedings X-ray Astronomy '999

- Stellar Endpoints, AGN and the Diffuse Background, ed. G. Malaguti, G. Palumbo, \& N. White, AIP Series of Conference Proceedings, in press [astro-ph/9911338]

Strohmayer, T. E., \& Brown, E. F. 2001, ApJ, in press [astro-ph/0108420]

Sugimoto, T., Ebisuzaki, T., \& Hanawa, T. 1984, PASJ, 36, 839

Swank, J. H., Becker, R. H., Boldt, E. A., et al. 1977, ApJ, 212, L73

Sztajno, M., van Paradijs, J., Lewin, W. H. G., et al. 1985, ApJ, 299, 487

Sztajno, M., van Paradijs, J., Lewin, W. H. G., et al. 1986, MNRAS, 222, 499

Taam, R. E., Woosley, S. E., \& Lamb, D. Q. 1996, ApJ, 459, 271

Tawara, Y., Hayakawa, S., \& Kii, T. 1984b, PASJ, 36, 845

Tawara, Y., Kii, T., Hayakawa, S., et al. 1984a, ApJ, 276, L41

Tawara, Y., Hirano, T., Kii, T., Matsuoka, M., \& Murakami, T. $1984 \mathrm{c}$, PASJ, 36, 861

Titarchuk, L. G. 1988, Sov. Astron. Lett., 14, 229

Titarchuk, L. 1994, ApJ, 429, 340

Thorsett, S. E., \& Chakrabarty, D. 1999, ApJ, 512, 288

Vaughan, B. A., van der Klis, M., Wood, K. S., et al. 1994, ApJ, 435, 362

van der Klis, M. 1989, in Timing Neutron Stars, ed. H. Ögelman, \& E. P. J. van den Heuvel (Kluwer Academic Publishers), 27

van der Klis, M. 2000, ARA\&A, 38, 717

van der Klis, M. 2001, ApJ, 561, 943

van Paradijs, J. 1982, A\&A, 107, 51

van Paradijs, J., Dotani, T., Tanaka, Y., \& Tsuru, T. 1990, PASJ, 42, 633

van Paradijs, J., \& Lewin, W. H. G. 1986, A\&A, 157, L10

van Paradijs, J., \& Lewin, W. H. G. 1987, A\&A, 172, L20

van Paradijs, J., Penninx, W., \& Lewin, W. H. G. 1988, MNRAS, 233, 437

van Straaten, S., van der Klis, M., Kuulkers, E., \& Méndez, M. 2001, ApJ, 551, 907

White, N. E., Peacock, A., Hasinger, G., et al. 1986, MNRAS, 218,129

Wijnands, R. 2001, ApJ, 554, L59

Wijnands, R. A. D., van der Klis, M., Kuulkers, E., Asai, K., \& Hasinger, G. 1997, A\&A, 323, 399

Woosley, S. E., \& Taam, R. E. 1976, Nature, 263, 101 